\title{
Optimizing the Yield of a Pure Enantiomer by Integrating Chiral SMB Chromatography and Racemization. Part 2: Theory
}

\section{Journal Article}

Author(s):

Breveglieri, Francesca; Otgonbayar, Tuvshinjargal (D); Mazzotti, Marco

Publication date:

2021-07-28

Permanent link:

https://doi.org/10.3929/ethz-b-000500366

Rights / license:

Creative Commons Attribution-NonCommercial-NoDerivatives 4.0 International

Originally published in:

Industrial \& Engineering Chemistry Research 60(29), https://doi.org/10.1021/acs.iecr.1c00680

\section{Funding acknowledgement:}

722456 - Continuous Resolution and Deracemization of Chiral Compounds by Crystallization (EC) 


\title{
Optimizing the Yield of a Pure Enantiomer by Integrating Chiral SMB Chromatography and Racemization. Part 2: Theory
}

\author{
Francesca Breveglieri, " Tuvshinjargal Otgonbayar," and Marco Mazzotti*
}

Cite This: Ind. Eng. Chem. Res. 2021, 60, 10720-10735

Read Online

ABSTRACT: We address the purification of the target enantiomer of a chiral compound from its racemic mixture, through simulated moving bed (SMB) chromatography either as a standalone or combined with the recycle and racemization of the undesired enantiomer. We carry out a comparative assessment of the two processes with focus on the role of the racemization kinetics, on the effect of the selectivity of the chiral stationary phase, and of the upstream symmetric synthesis of the racemate. The analysis is general thanks to the methods adopted for the optimal design of the integrated process (with racemization) and for the sizing of the SMB unit, as well as for the use of the four generalized Langmuir adsorption isotherms. In this way, we determine quantitative criteria that show that the standalone SMB

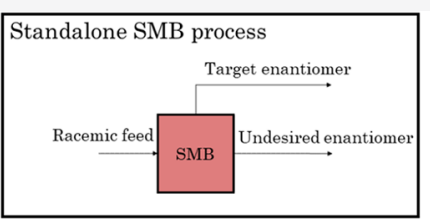

Integrated process

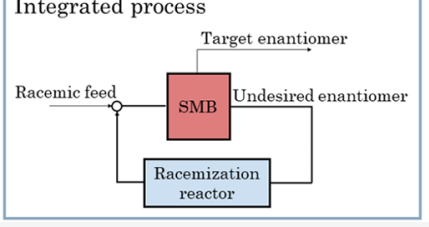

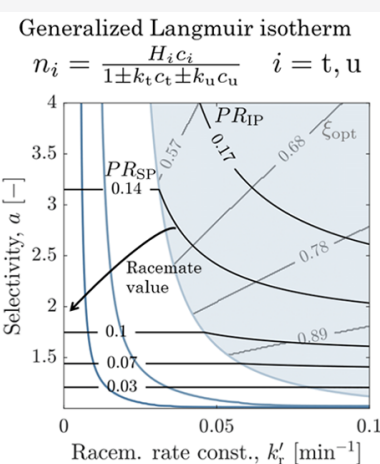
process outperforms the integrated process when the performance of the racemization catalyst or the chiral stationary phase is poor and the racemic feed is inexpensive.

\section{INTRODUCTION}

Obtaining the pure enantiomer of a chiral compound, for example, of a pharmaceutical, an agro-chemical, or a nutritional product, requires either enantioselective synthesis, ideally achieving $100 \%$ yield though more expensive, or symmetric synthesis yielding a 50/50 racemate followed by chiral separation of the target enantiomer (eutomer) from the undesired distomer. ${ }^{1}$ Such combination attains $50 \%$ yield when the distomer is wasted, and can in principle achieve $100 \%$ yield if the distomer undergoes racemization and the product is recycled. Possible separation techniques are based on chiral chromatography, both batch and continuous, such as simulated moving bed (SMB) chromatography. Alternatively, crystallization offers the possibility of combining separation and racemization in a single step; examples are either preferential crystallization $^{2-4}$ or solid-state deracemization, the latter through Viedma ripening or via temperature cycles. ${ }^{5-9}$

In this contribution, we focus on the combination of symmetric synthesis and multicolumn continuous chiral chromatography using SMB either with or without recycle and racemization of the distomer. We are obviously inspired by the characterization study reported in part 1 of this series. ${ }^{10}$ Though we are not the first to analyze and compare these two alternatives, we observe that in the previous literature whenever racemization and recycle have been considered, the racemization reaction has always been assumed to reach equilibrium. $^{11-16}$ Such assumption has the important implication that the outlet of the racemization reactor is a racemate, which-when mixed with the racemic feed to the overall process-yields an SMB feed that is always racemic. On the one hand this greatly simplifies the comparative assessment of a standalone SMB process (SP in the following) and an integrated SMB and racemization process (IP below), but on the other hand it weakens the relevance of the analysis itself. Our own experience indicates that finding an effective racemization catalyst is very challenging, ${ }^{10}$ particularly when there is the constraint that the chromatographic mobile phase and the racemization solvent are one and the same. Therefore, in many cases, a meaningful comparative study of standalone and integrated processes needs to account for the possibility of an incomplete racemization reaction, hence of a nonracemic feed to the SMB. A similar situation was studied by Kaspereit et al. in an SMB-crystallization process without a racemization step, where the occurrence of a nonracemic SMB feed is a consequence of recycling the mother liquor from the crystallization step. To account for the SMB feed composition in the process performance evaluation, they developed a simple shortcut model that is based on principles also used here. In this work, the shortcut approach is further improved and

Special Issue: Giuseppe Storti Festschrift

Received: February 15, 2021

Revised: May 1, 2021

Accepted: May 2, 2021

Published: May 12, 2021 
adapted to the analysis of processes with the racemization step as a bottleneck. ${ }^{17}$

The fundamental question that this work addresses is which of the two processes, i.e. SP and IP, is better, and when and why this is the case. There are at least three factors that play a role in this context. First, if the racemate is inexpensive then wasting the distomer after the SMB separation is immaterial; hence, the SP will be better than the IP (this effect has already been studied $\left.{ }^{11,12}\right)$. Second, if the selectivity and separation efficiency of the chiral stationary phase used in the SMB are poor, then the IP-which through recycling separates molecules of the two already separated enantiomers again and again-cannot be favored. Finally, if the racemization reaction rate is low, then again, recycling is not attractive and the SP will be preferred. It follows that there must be a threshold in the parameter space of these processes that separates conditions where the SP is preferred from conditions where the opposite is true. It is such a threshold that we are seeking.

The key novelty of this work is that we can determine such threshold conditions in the most general case, considering the effects of the "cost" of the upstream (symmetric) synthesis step, and of the chromatographic selectivity, as well as the effect of the rate of the racemization reaction. The challenge is that for every set of the system's features, the two processes have to be optimized so that their performance can be compared with each other and with the performances obtained for any other set of features. Such optimization is straightforward from a conceptual perspective but rather challenging computationally, unless very carefully selected simplifications are made and shortcuts are taken, as discussed in detail in the paper.

Thus, for instance, although it is well-known that any physically plausible binary adsorption isotherm leads, whatever the feed composition, to a complete separation region in the space of the SMB's operating conditions that has similar topological features as any other, ${ }^{18}$ the selection of the optimal operating point for a specific feed composition for such an isotherm would be extremely time-consuming. It is for this reason that we have confined ourselves to the four generalized Langmuir isotherms only, because they exhibit quite diverse behavior while their corresponding optimal operating parameter values are known exactly for any feed composition (see Mazzotti $^{19}$ for the detailed derivations, Mazzotti ${ }^{20}$ for a comprehensive collection of all the equations, and the review by Rajendran et al. ${ }^{18}$ for a summary of the key concepts and equations).

A further complication results from the necessity to establish a general enough criterion for the comparative assessment of the two processes. It is quite clear that the comparison must account for the possibility, or better the certainty, that the optimal SMB unit in the SP case (no recycle, 50\% yield for the overall process) is different than the one in the IP case (with recycle and $100 \%$ yield). For this to be possible the SMB optimization cannot be carried out for a specific geometry, but it has to include the column size (length and diameter) among the decision variables. This hurdle was overcome by implementing a shortcut method based on physical constraints on the system, similarly proposed in previous work. ${ }^{17}$

The paper is organized as follows. In section 2 we provide the preliminaries, including assumptions and simplifications. In section 3 we present the model equations for the two processes, as well as the criteria for their performance assessment. The results of the comparative analysis of the two processes for the four generalized Langmuir isotherms are reported in section 4 . In this section, first, the basis for the comparison and the constraints for the application of the triangle theory are discussed, then, the effect of the racemization rate on the IP performance is described. Next, the determination of the threshold separating regions in the space of the system's features where one process is preferred over the other is presented. Finally, section 5 is dedicated to the analysis of the effect of other design parameters, such as the column efficiency and the purity requirements. This is followed by conclusions (section 6).

\section{PRELIMINARIES}

2.1. Simulated Moving Bed Chromatography. An SMB unit consists of a number of chromatographic columns, grouped in four sections, indexed from $j=1$ to 4 , which are defined by the position of the four inlet (desorbent stream, labeled D, consisting of the mobile phase, and feed, F, containing the two enantiomers to be separated) and outlet (extract, E, where the more retained enantiomer is collected, and raffinate, $\mathrm{R}$, where the less retained enantiomer is withdrawn) ports. Such ports move periodically and synchronously in the direction of the fluid flow (of a distance equal to one column length) so as to simulate the countercurrent movement of the solid phase (which is in fact fixed). Successive port switches are separated by a time interval called switch time, $t^{*}$. The desorbent enters before section 1 ; the extract and the raffinate are collected between sections 1 and 2 and between sections 3 and 4, respectively; the feed enters between sections 2 and 3 . The separation of the two enantiomers is controlled by how sections 2 and 3 are operated and where it takes place, provided in section 1 the solid phase is regenerated and in section 4 the mobile phase is cleansed of all the solutes before leaving the last column of section 4 and being recycled to the inlet of the first column of section 1. Operation and separation performance are controlled by choosing wisely the volumetric flow rates in the four SMB sections $\left(Q_{j}\right.$, with $\left.j=1, \ldots, 4\right)$ and the switch time, $t^{*}$. On the basis of the description above, the following material balances apply at the inlet and outlet ports:

$$
\begin{aligned}
& Q_{D}=Q_{1}-Q_{4} \\
& Q_{E}=Q_{1}-Q_{2} \\
& Q_{F}=Q_{3}-Q_{2} \\
& Q_{R}=Q_{3}-Q_{4}
\end{aligned}
$$

together with the corresponding component balances for the two enantiomers. A comprehensive review of SMB for chiral separations, containing all the concepts and information needed here, can be consulted by the interested reader. ${ }^{18}$ It is worth noting that though different variants of the standard SMB operation presented here exist (see the same review for details), they are immaterial for this study and will not be mentioned any further.

There are shortcut criteria to choose SMB operating conditions that were developed based on the so-called equilibrium theory of nonlinear chromatography. ${ }^{18,19,21,22}$ Such an approach hinges on the key operating variables, which are the dimensionless flow rate ratios, $m_{j}$, with $j=1, \ldots$, 4. These are defined for each SMB section as the ratio of the 


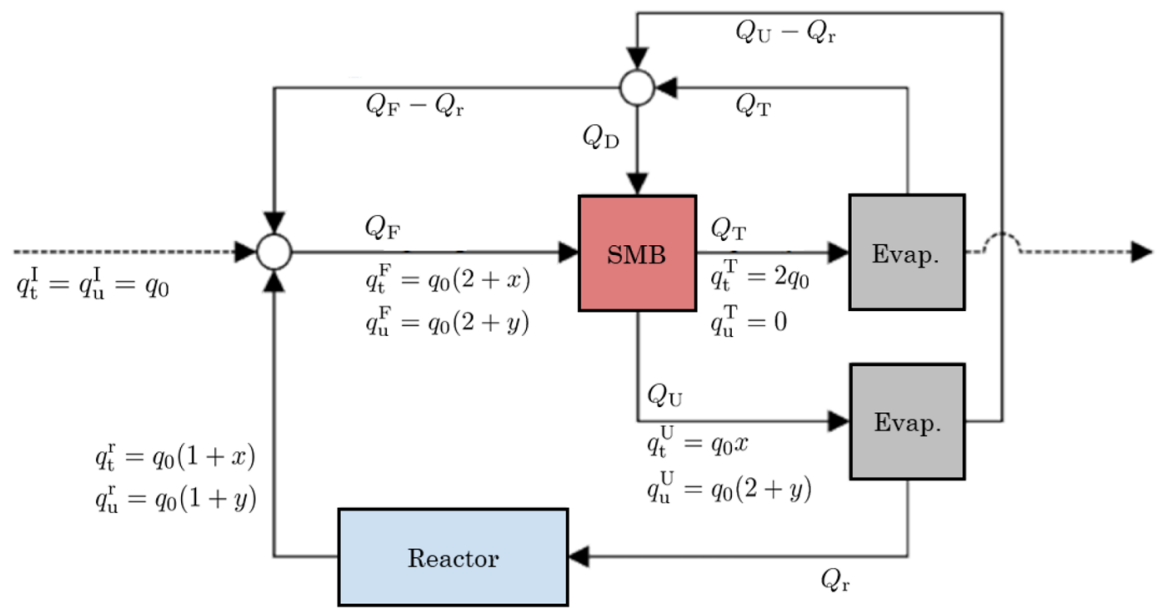

Figure 1. Flowsheet of the integrated process.

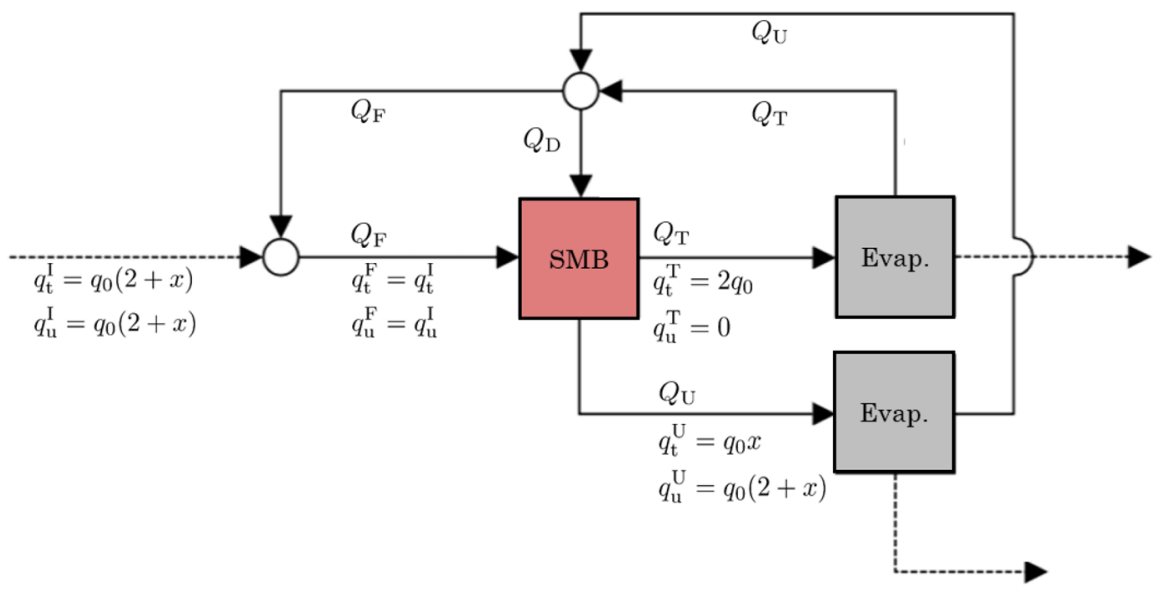

Figure 2. Flowsheet of the standalone SMB process.

net fluid flow rate and the simulated solid flow rate, and combine operating and geometric parameters:

$$
m_{j}=\frac{Q_{j} t^{*}-V \epsilon^{*}}{V\left(1-\epsilon^{*}\right)}
$$

where $V=A L$ is the column volume ( $A$ and $L$ are column cross-section and length, respectively) and $\epsilon^{*}$ is the overall bed void fraction, including both intra- and interparticle voids. It has been possible to show that the values of the four flow rate ratios are constrained and must belong to given intervals for the separation to be complete, that is, for the extract and the raffinate to contain one and only one of the two components to be separated. The bounds defining such intervals depend on the adsorption isotherm, to which the system is subjected, and on the feed composition. They can be calculated exactly for all the four generalized Langmuir isotherms, including the operating point where separation performance is optimal. ${ }^{19,20}$ The complete separation region in the operating parameter space can be effectively visualized (see previous references, as well the first part of this series ${ }^{10}$ ), and the whole approach has been dubbed triangle theory because of the shape of the complete separation region in the $\left(m_{2}, m_{3}\right)$ plane in the case of the most common isotherms.

While triangle theory allows for choosing the values of $m_{1}$, $m_{2}, m_{3}$, and $m_{4}$, it accounts for neither pressure drop limitations nor column efficiency. Hence it cannot be used to choose the value of $t^{*}$; actually in the scope of triangle theory productivity is inversely proportional to $t^{*}$ hence the optimal value of $t^{*}$ would in principle be as small as possible, which would imply infeasible and infinitely large flow rates to keep the values of $m_{j}$ constant according to eq 2 . In practice, the switch time can be determined when one accounts for pressure drop, which increases linearly with the flow rate, and for column efficiency, which decreases with the flow rate and is typically assessed through detailed simulations. This approach would not bode well in the context of the broad parametric analysis that we want to carry out here, thus rather conservative values will be chosen to ensure the legitimate application of the shortcut approach as discussed in section 5 . Moreover, it is worth noting, that if the calculated switch time values are too low to be physically feasible, one can lower the $\Delta P_{\max }$ constraint accordingly so as to increase $t^{*}$.

2.2. Solubility. The chiral compounds of interest here are solid, or liquid at ambient conditions (for the separation of gaseous enantiomers by SMB see previous literature ${ }^{23-25}$ ). As a consequence, solid-liquid equilibrium is important, as shown also in the first part of this series. ${ }^{10}$ Phase diagrams involving the enantiomers of a chiral compound in an achiral solvent are symmetric. While the solubility of each enantiomer may increase or decrease when the concentration of the counterenantiomer increases, it is not uncommon that such an effect is minor. $^{26,27}$ We will make such an assumption in the scope of 
this study. As a consequence, the solubility of each enantiomer at the system temperature is the same, and it is constant, namely $c^{*}$, that is, independent of the concentration of the counter-enantiomer. This concentration cannot be exceeded anywhere in the process streams, particularly in the SMB feed and in the two outlet streams, that is, extract and raffinate, in order to avoid any undesired precipitation.

2.3. Racemization. Racemization is a reversible catalytic reaction that converts one enantiomer into the other. ${ }^{28}$ At chemical equilibrium, the system composition is racemic, that is, $50 \%$ of each enantiomer. The reaction can occur spontaneously or with the aid of either a homogeneous or a heterogeneous catalyst that is immobilized on a solid support in a fixed bed reactor (PFR, plug flow reactor). The latter is the best solution in the context of this study because it minimizes issues in the recycle of the racemization products and losses of catalyst. To describe the evolution of the relative concentration of the two enantiomers the same equation can be used for a batch reactor, with both homogeneous and heterogeneous catalysts, and for a fixed bed reactor, assuming it is a plug flow reactor (PFR), where time, $\tau$, represents physical time in the former and residence time in the latter:

$$
\ln e e(\tau)=\ln e e_{0}-2 k_{\mathrm{r}}^{\prime} \tau
$$

where $e e$ is the enantiomeric excess, defined as ee $=\left(c_{\mathrm{u}}-c_{\mathrm{t}}\right) /$ $\left(c_{\mathrm{u}}+c_{\mathrm{t}}\right)$ (with the subscripts $\mathrm{u}$ and $\mathrm{t}$ indicating the undesired and the target enantiomer, respectively), and $e e_{0}$ is its initial value in the batch case and its inlet value in the PFR case; $k_{\mathrm{r}}^{\prime}$ is the racemization rate constant, which depends on the temperature and on the catalyst concentration. ${ }^{10,28,29}$

\section{METHODOLOGY FOR THE ANALYSIS OF TWO PROCESS ALTERNATIVES}

3.1. Process Schemes and Notation. Figures 1 and 2 show the schemes of the integrated process, IP, and of the standalone SMB process, SP, respectively. Before analyzing the two processes in detail it is worth explaining the notation that is utilized in this work and in both figures. In the SMB literature that we refer to, the SMB streams are labeled as in eqs 1 , and the two species to be separated are labeled with the indices 1 (or B) and 2 (or A), where species 2 is more retained than species 1 .

This standard notation is not sufficient in the context of this work because the choice of the target enantiomer is completely unrelated to the retention order exhibited by the chiral stationary phase used in the SMB. We have therefore chosen to label the desired eutomer with the small letter "t", for target, and the undesired distomer with "u", for undesired, irrespective of which of the two is more retained. Accordingly, the stream where the eutomer is withdrawn is labeled with the capital letter " $\mathrm{T}$ ", for target, and that where the distomer is withdrawn with "U", for undesired, irrespective of which of the two is the extract. Such unconventional notation complies with obvious rules, namely that if $\mathrm{t}=2$ and $\mathrm{u}=1$, then $\mathrm{T}=\mathrm{E}$ and $\mathrm{U}=\mathrm{R}$; on the contrary if $\mathrm{t}=1$ and $\mathrm{u}=2$, then $\mathrm{T}=\mathrm{R}$ and $\mathrm{U}=\mathrm{E}$. We will have to distinguish between these two cases when presenting the results of the analysis, but not when writing the model equations and when discussing optimization. Moreover, the relevant quantities at the racemization reactor outlet and inlet are labeled with the lowercase $r$, whereas the feed to the whole process is labeled as I.

We assume that the whole process is carried out at a constant temperature, thus the physicochemical parameters of the different units are chosen at the corresponding temperature. Moreover, pressure is immaterial, except in the evaporators, which are anyways treated as ideal separators. Any process stream is characterized not only by its volumetric flow rate, $Q$ and the enantiomers' concentration, $c_{i}$, but also by the molar flow of each enantiomer, $q_{i}=Q c_{i}$. The last quantity will play a key role, and will be sometimes loosely referred to as the "amount" of the ith enantiomer in the specified stream.

Both processes will be designed and optimized for a specified amount of eutomer produced, namely $2 q_{0}$, with $q_{0}$ being the amount chosen as basis for comparison.

3.2. Solute Balances. Integrated Process. When inspecting Figure 1, it might not appear obvious that the concentrations of the two enantiomers in the different process streams depend on only two degrees of freedom, that is, on two parameters with a clear physical meaning. This becomes apparent when one considers both process constraints and specifications (note that the solutes' molar flows before and after the evaporators are by definition the same). Since the target stream must be pure and the overall feed is a racemate, then $q_{\mathrm{t}}^{\mathrm{T}}=2 q_{0}$ and $q_{\mathrm{u}}^{\mathrm{T}}=0$, and $q_{\mathrm{t}}^{\mathrm{I}}=q_{\mathrm{u}}^{\mathrm{I}}=q_{0}$. This implies that an amount $q_{0}$ of the distomer must be racemized. Then, the eutomer's amount in the recycled stream must be at least $q_{0}$; hence, $q_{\mathrm{t}}^{\mathrm{r}}=q_{0}(1+x)$, with $x$ being a non-negative quantity. It follows immediately that $q_{\mathrm{u}}^{\mathrm{r}}=q_{0}(1+y)$, with $y \geq x \geq 0$, because the recycled stream can either be enriched in the distomer (if $y>x$ ), or be racemic (if $y=x$ ). From the outlet of the racemization reactor, calculating material balances downstream, in the SMB feed, yields $q_{\mathrm{u}}^{\mathrm{F}}=q_{0}(2+y)$ and $q_{\mathrm{t}}^{\mathrm{F}}=q_{0}(2+$ $x)$, while calculating them upstream, in the $\mathrm{U}$ stream, leads to $q_{\mathrm{u}}^{\mathrm{U}}=q_{0}(2+y)$ and $q_{\mathrm{t}}^{\mathrm{U}}=q_{0} x$.

When inspecting Figure 1 again after these considerations, on the one hand it is apparent that the parameter $x$ is proportional to the amount of the eutomer that leaks in the SMB through the wrong outlet stream because of incomplete separation conditions. Note that $x$ is a design variable in our study, and in this context, we will set $x=0$ for the analysis carried out in the first part of the manuscript, as it allows for a direct determination of the operating conditions via triangle theory. We will later show how this choice barely affects the trends identified in our study (see section 5.2). On the other hand, the parameter $y$ gives the amount of the distomer in excess that keeps recirculating through the IP. Its important role is highlighted when the relative conversion $\xi$ is defined, that is, the ratio between the actual and the maximum theoretical conversion of the racemization reaction:

$$
\xi=\frac{2\left(q_{\mathrm{u}}^{\mathrm{U}}-q_{\mathrm{u}}^{\mathrm{r}}\right)}{q_{\mathrm{u}}^{\mathrm{U}}-q_{\mathrm{t}}^{\mathrm{U}}}=\frac{2}{2+y-x}
$$

To deliver the extent of racemization needed, when $y=x$ (i.e., when $y=0$ in the case where $x=0$ ) the relative conversion must be $\xi=1$; hence, the residence time is infinite. Whenever $y$ $>x$, then $\xi<1$ and the desired conversion is attained in a finite time. Therefore, the parameter $y$ is a key degree of freedom in the design and a key decision variable in the optimization of the IP. Note that both $x$ and $y$ are virtually unbounded quantities.

Standalone Process. With neither racemization nor recycle, the amount of the eutomer to be delivered in the corresponding outlet stream of the SMB must be already present in the overall process, which is by definition racemic. As a consequence, the following relationships can be easily 
established depending on a single degree of freedom, $x$ (see Figure 2): $q_{\mathrm{t}}^{\mathrm{T}}=2 q_{0}$ and $q_{\mathrm{u}}^{\mathrm{T}}=0 ; q_{\mathrm{t}}^{\mathrm{I}}=q_{\mathrm{t}}^{\mathrm{F}}=q_{0}(2+x)=q_{\mathrm{u}}^{\mathrm{I}}=q_{\mathrm{u}}^{\mathrm{F}}$; $q_{\mathrm{t}}^{\mathrm{U}}=q_{0} x$ and $q_{\mathrm{u}}^{\mathrm{U}}=q_{0}(2+x)$. It follows that the only reason not to have $x=0$ is if one aims to operate the SMB at incomplete separation conditions, which could give some advantages in certain cases in terms of higher productivity. In this work, we will (mostly) focus on the case $x=0$.

3.3. Sizing Process Units. Sizing the SMB Unit. Sizing the SMB unit for a specified value of $y$ and for $x=0$ requires first of all determining the flow rate ratios, $m_{j}$, through triangle theory, which in turn requires knowing the SMB feed composition. On the basis of this information, triangle theory allows for calculating the optimal $m_{j}$ values, and choosing their operating values (see section 2.1). In the following, for simplicity but without loss of generality, we will always use the optimal values of the $m_{j}$ parameters obtained for the adsorption isotherm of interest and the selected feed composition.

The criterion for the choice of the SMB feed composition is that of operating at the highest concentration possible, because this is the conditions with which the SMB unit attains its optimal productivity. There are cases in which the adsorption isotherm exhibits a very strong nonlinearity and it might be advisible to operate at a lower concentration. Nevertheless, in the following, we choose the solvent flow rate of the stream for which the concentration of one enantiomer is the highest so that the concentration is equal to a set value as close as possible to the solubility limit, $c^{*}$, that is, separated from it by a reasonable safety margin. For the sake of simplicity, and without loss of generality, in the following, we assume that the safety margin is zero and that the maximum concentration mentioned above is indeed $c^{*}$. However, we cannot know $a$ priori in which stream and for which enantiomer such a critical condition occurs.

On the basis of these considerations and on the fact that the optimal flow rate ratios, $m_{j}$, depend on the feed composition, the design procedure proposed here starts by assigning a value to the SMB feed concentration of the distomer, $c_{\mathrm{u}}^{\mathrm{F}}$. Such a concentration will be equal to $c^{*}$, if that concentration is the highest in the whole flow sheet. When this is not the case, $c_{u}^{\mathrm{F}}$ must be reduced following the criteria discussed at the end of this subsection. Whatever the selected feed concentration, the SMB feed flow rate, $Q_{F}$, must be calculated as follows (scaled with respect to the quantity $q_{0}$ ):

$$
\frac{Q_{\mathrm{F}}}{q_{0}}=\frac{2+y}{c_{\mathrm{u}}^{\mathrm{F}}}
$$

Then the feed concentration of the eutomer is $c_{\mathrm{t}}^{\mathrm{F}}=c_{\mathrm{u}}^{\mathrm{F}}(2+x) /$ $(2+y)$. Having determined the SMB feed composition, the values of the flow rate ratios, $m_{1}, m_{2}, m_{3}$, and $m_{4}$, can be assigned from the triangle theory. Note that eq 5 is written with reference to the IP of Figure 1. If the SP of Figure 2 is considered instead, then in the last eq $(2+y)$ would be substituted by $(2+x)$, and everything that follows would change accordingly.

Using the value of $Q_{F}$ just calculated, the node balance of eq $1 c$ and the definition of $m_{j}$ in eq 2 allows establishing the following constraint on the ratio of column volume, $V$, and switch time, $t^{*}$ :

$$
\frac{Q_{\mathrm{F}} t^{*}}{V}=\left(m_{3}-m_{2}\right)\left(1-\epsilon^{*}\right)
$$

Then, the last equation can be used to eliminate such a ratio from all $m_{j}$ values, thus obtaining the internal SMB flow rates in terms of $Q_{F}$ and the flow rate ratios:

$$
\frac{Q_{j}}{q_{0}}=\frac{m_{j}\left(1-\epsilon^{*}\right)+\epsilon^{*}}{\left(m_{3}-m_{2}\right)\left(1-\epsilon^{*}\right)} \frac{Q_{F}}{q_{0}} \quad(j=1, \ldots, 4)
$$

There remain three quantities to be calculated, namely the individual values of $V$ and $t^{*}$, plus the column length $L$. These must obey eq 6 plus two conditions that are normally assigned as inequalities, namely an upper bound on the total pressure drop, $\Delta P_{\max }$ and a lower bound on the number of theoretical stages, $N_{\min }$ in the SMB. The former can be assigned by assuming a proportionality relationship (Darcy's law) between pressure drop per unit length and fluid velocity:

$$
\frac{\Delta P}{L}=\frac{\lambda Q}{A}
$$

The latter can be dealt with by assuming a simplified version of the van Deemter equation, which allows us to obtain explicit expressions for the unit design equations, without loss of generality. This is given by the following proportionality relationship between height equivalent to a theoretical stage and fluid velocity:

$$
\frac{L}{N}=\frac{h Q}{A}
$$

In this simplified description of pressure drop and efficiency in the chromatographic columns used in the SMB, the parameters $\lambda$ and $h$ are the proportionality constants, which can be easily estimated from ad hoc experiments.

Considering an SMB plant where each section has $n_{j}$ columns $(j=1, \ldots, 4)$ and using eqs 8 and 9 yield the following constraints:

$$
\begin{aligned}
& \Delta P_{\max } \geq \frac{\lambda L^{2}}{V} \sum_{j=1}^{4} n_{j} Q_{j}=\frac{\lambda L^{2} Q_{\mathrm{F}} \sigma_{\mathrm{N}}}{V\left(m_{3}-m_{2}\right)\left(1-\epsilon^{*}\right)}, \\
& N_{\min } \leq \frac{V}{h} \sum_{j=1}^{4} \frac{n_{j}}{Q_{j}}=\frac{\sigma_{\mathrm{D}} V\left(m_{3}-m_{2}\right)\left(1-\epsilon^{*}\right)}{h Q_{\mathrm{F}}},
\end{aligned}
$$

where the parameters $\sigma_{\mathrm{N}}$ and $\sigma_{\mathrm{D}}$ are defined using eq 7 as follows:

$$
\begin{aligned}
& \sigma_{\mathrm{N}}=\sum_{j=1}^{4} n_{j}\left(m_{j}\left(1-\epsilon^{*}\right)+\epsilon^{*}\right), \\
& \sigma_{\mathrm{D}}=\sum_{j=1}^{4} \frac{n_{j}}{m_{j}\left(1-\epsilon^{*}\right)+\epsilon^{*}}
\end{aligned}
$$

Decreasing the ratio $Q_{F} / V$, which is proportional to the productivity, that is, the amount processed per unit time and volume, would make fulfilling the last two constraints easier but with a penalization of performance. Hence, we have decided to size the SMB so as to fulfill eq 6, as well as the two inequalities of eqs 10 and 11 , with the two right-hand side expressions attaining the upper and lower bound, respectively, of the corresponding inequality. Enforcing these three constraints allows calculating exactly the three missing quantities $V, L$, and $t^{*}$ : 


$$
\begin{aligned}
\frac{V}{q_{0}} & =\frac{h N_{\min }}{\sigma_{\mathrm{D}}\left(m_{3}-m_{2}\right)\left(1-\epsilon^{*}\right)} \frac{Q_{\mathrm{F}}}{q_{0}} \\
L^{2} & =\frac{h N_{\min } \Delta P_{\max }}{\lambda \sigma_{\mathrm{N}} \sigma_{\mathrm{D}}} \\
t^{*} & =\frac{h N_{\text {min }}}{\sigma_{\mathrm{D}}}
\end{aligned}
$$

It is worth noting that the column volume is also scaled with $q_{0}$, like the flow rates.

Let us now consider the issue mentioned when introducing eq 5 , that is, the issue of where in the process flow sheet the highest concentration occurs, when not in the SMB feed stream; in that position the solubility constraint must be activated. It is worth restating that eq 5 determines the feed flow rate such that the distomer concentration equals the fixed value $c_{\mathrm{u}}^{\mathrm{F}}$. With this, the composition of the SMB feed is known and through triangle theory all the flow rate ratios, $m_{j}(j=1, \ldots$, $4)$, can be selected.

Note that in any given stream the $i$ th solute concentration is given by the ratio between solute molar flow and flow rate, that is, $c_{i}=q_{i} / Q$. Thus, when the $m_{j}$ definition of eq 2 is combined with the enantiomer molar flows reported in Figures 1 and 2 and the node balances of eq 1 , the following four concentration ratios that compare the distomer's concentration in the feed with the solute's concentration in either the extract or raffinate can be calculated:

$$
\begin{gathered}
\frac{c_{\mathrm{u}}^{\mathrm{F}}}{c_{\mathrm{u} / \mathrm{t}}^{\mathrm{E}}}=\frac{\left(m_{1}-m_{2}\right) q_{\mathrm{u}}^{\mathrm{F}}}{\left(m_{3}-m_{2}\right) q_{\mathrm{u} / \mathrm{t}}^{\mathrm{E}}} \\
\frac{c_{\mathrm{u}}^{\mathrm{F}}}{c_{\mathrm{u} / \mathrm{t}}^{\mathrm{R}}}=\frac{\left(m_{3}-m_{4}\right) q_{\mathrm{u}}^{\mathrm{F}}}{\left(m_{3}-m_{2}\right) q_{\mathrm{u} / \mathrm{t}}^{\mathrm{R}}}
\end{gathered}
$$

If all four ratios are larger than 1 , then the SMB feed stream is the critical one, and therefore $c_{\mathrm{u}}^{\mathrm{F}}$ should be chosen equal to $c^{*}$. If any of the four ratios is smaller than 1 , then that enantiomer in that outlet stream is the critical component and its concentration in that stream should be $c^{*}$. To achieve that, then the distomer's concentration in the feed used in eq 5 should be reduced-possibly in an iterative procedure-until the mentioned critical concentration reaches the target value $c^{*}$.

Sizing the Racemization Reactor. Let us consider the IP in Figure 1, particularly the racemization reactor. Substituting the streams' compositions shown in the figure into the definition of the enantiomeric excess and using the relationship for $\xi$ from eq 3 and eq 4 can be written as

$$
2 k_{\mathrm{r}}^{\prime} \tau_{\mathrm{r}}=\ln \left(\frac{2+y-x}{y-x}\right)=-\ln (1-\xi)
$$

with the residence time $\tau_{\mathrm{r}}=V_{\mathrm{r}} \epsilon_{\mathrm{r}} / Q_{\mathrm{r}}$, where $V_{\mathrm{r}}$ and $\epsilon_{\mathrm{r}}$ are the packed bed reactor's volume and void fraction, respectively, and $Q_{r}$ is the volumetric flow rate through the reactor. Our design criterion is that of minimizing the reactor volume for a given residence time and relative conversion. This entails minimizing the flow rate $Q_{r}$, hence enforcing the condition that the inlet distomer's concentration $c_{\mathrm{u}}^{\mathrm{r}}$ be equal to $c^{*}$. Then $Q_{r}=$ $q_{0}(2+y) / c_{\mathrm{w}}^{\mathrm{r}}$, and the reactor volume from eq 18 is (scaled with respect to $q_{0}$ ):

$$
\frac{V_{\mathrm{r}}}{q_{0}}=-\frac{(2+y) \ln (1-\xi)}{2 k_{\mathrm{r}}^{\prime} \epsilon_{\mathrm{r}} c_{\mathrm{u}}^{\mathrm{r}}}
$$

Solvent Balances and Sizing of the Evaporators. The balances of the solvent (in chromatography: the mobile phase) is important for the sizing of the evaporators. In this work, each evaporator is treated as an ideal unit that removes whichever specified amount of solvent, $Q_{\mathrm{v}}$ in a fixed residence time $\tau_{\mathrm{v}}$. The size of the evaporator is given by the corresponding volume, that is, $V_{\mathrm{v}}=Q_{\mathrm{v}} \tau_{\mathrm{v}}$.

The case of the SP in Figure 2 is rather simple, because the two evaporators evaporate all the solvent, thus separating it from the dry enantiomeric solids. Therefore, the solvent flow rates before and after the two evaporators are identical, their sum being $Q_{T}+Q_{U}=Q_{D}+Q_{F}$. These two streams are mixed, condensed, then split into the solvent stream for the SMB feed, $Q_{F}$ (to dissolve the racemic feed to the SP, assumed to consist of a dry solid phase, and to convey it to the SMB) and into the desorbent stream, $Q_{D}$.

The case of the IP in Figure 1 is slightly more complex. On the one hand, the evaporator on the $Q_{T}$ stream behaves in the same way, as it delivers the dry pure product and a solvent stream with flow rate $Q_{T}$. On the other hand, the $Q_{U}$ stream goes through a partial evaporator that evaporates the solvent at a rate given by the difference $Q_{U}-Q_{r}$. The solvent constituting the stream through the racemization reactor, with flow rate $Q_{r}$, is directly recycled into the SMB feed stream. The two solvent streams from the two evaporators, are mixed, condensed, and split into the desorbent stream and a solvent stream, which reunites with the recycled stream and together with the dry solid racemic feed to the IP constitutes the SMB feed, of flow rate $Q_{F}$. Depending on the flow rates attained in each specific case, and on whether the distomer's concentration in the feed is higher than its concentration in the undesired stream, of flow rate $Q_{U}$, it might happen that this second evaporator is not needed and can be omitted.

Sizing the Upstream Synthesis Process. The upstream section of the plant, where the symmetric synthesis of the racemate is carried out, needs to be sized so as to assess and quantify the relative merits of the IP with respect to the SP, due to the difference in the amounts of the racemate feed to either process. In this work, the size (volume) of the different process units, that is, SMB, reactor, and evaporators, is considered, therefore accordingly also an estimate of the volume of the upstream synthesis section is calculated. Such volume, $V_{\text {up }}$, is assumed to be equal to the product of three quantities, namely: (i) the molar flow of racemate, which is $2 q_{0}$ in the case of the IP and $2(2+x) q_{0}$ in the case of the SP, i.e. at least twice as much; (ii) the average residence time of the racemate in the synthesis section, $\tau_{\text {up }}$ (iii) the average volume needed for the production of one unit of racemic compound, called $v_{\text {up }}$. This assumption results in the following formula:

$$
V_{\text {up }}=v_{\text {up }} \tau_{\text {up }}\left(q_{\mathrm{t}}^{\mathrm{I}}+q_{\mathrm{u}}^{\mathrm{I}}\right)
$$

The effect of the residence time $\tau_{\text {up }}$ (of the order of days) will be assessed through parametric analysis. For the specific volume instead we make the drastic assumption that for a liquid phase process it is proportional to the reciprocal of the racemate solubility: hence $v_{\text {up }}=1 /\left(2 c^{*}\right)$.

3.4. Performance Assessment. The objective of this section is to define the performance indicator that allows a comparison of the IP and the SP process. In general, 
productivity is used, which is expressed as molar or mass throughput per unit time and volume of the equipment under consideration. The key pieces of equipment considered in this study are the SMB unit, the racemization reactor, the evaporators, and the upstream section where symmetric synthesis occurs. While such volumes have been calculated as described in the previous section, admittedly they cannot be considered equivalent. For instance, the SMB volume and the reactor volume are proportional to the amount of expensive chiral stationary phase and racemization catalyst, respectively; evaporators are obviously cheaper per unit volume, while the upstream section can be more or less expensive. To account for these effects, to each unit of type $k$, a unitary cost, $C_{k}$, and a lifespan, $l_{k}$ are associated, similarly to what was previously done in the literature. ${ }^{11,12}$ The cost per unit volume and unit time, $\pi_{k}$, is then obtained as $\pi_{k}=C_{k} / l_{k}$. Since we are interested in relative rather than absolute values, the normalized quantity $w_{k}$ $=\pi_{k} /\left(\sum_{i} \pi_{i}\right)$ is introduced. This is used as weight for the volume $V_{k}$ in the calculation of productivity, which is defined as

$$
P=\frac{2 q_{0}}{w_{\mathrm{SMB}} V_{\mathrm{SMB}}+w_{\mathrm{r}} V_{\mathrm{r}}+w_{\mathrm{v}} V_{\mathrm{v}}+w_{\mathrm{up}} V_{\mathrm{up}}}
$$

where $V_{\mathrm{r}}=0$ in the case of the SP.

Table 1 reports the reference values of the cost parameters used in this work (MU indicates a generic monetary unit).

Table 1. Estimated Values, $\pi$, of the Cost per Volume, $C$, and Life Span, $l$, of the Different Units, $k$, and Respective Normalized Value, $w$

$\begin{array}{lcccl}\text { process unit, } k & C_{k}\left[\mathrm{MU} \mathrm{mL}^{-1}\right] & l_{k}[\mathrm{~d}] & \pi_{k}\left[\mathrm{MU} \mathrm{mL} \mathrm{m}^{-1} \mathrm{~d}^{-1}\right] & w_{k}[-] \\ \text { SMB } & 100 & 1000 & 0.1 & 0.455 \\ \text { reactor (r) } & 10 & 100 & 0.1 & 0.455 \\ \text { evaporator (v) } & 10 & 1000 & 0.01 & 0.0455 \\ \text { upstream (up) } & 10 & 1000 & 0.01 & 0.0455\end{array}$

Note that a detailed economic analysis is out of the scope of the work, hence, these values are chosen based on common knowledge and educated guesses, so as to identify general trends rather than to draw specific conclusions. For this reason, a sensitivity analysis of the cost factors, where we vary them by orders of magnitude, is carried out and is presented.

\section{COMPARATIVE ASSESSMENT OF THE IP AND SP PROCESSES}

4.1. Basis for the Comparison. The overarching goal of this work is to provide quantitative criteria to decide for any specific system whether the integrated process, IP, is better than the standalone SMB process, SP, or not. This should be based on their productivity as defined in the previous section, which allows in principle for a techno-economic comparison. The challenge is that the processes, particularly the IP, are rather complex, their performance depending on a large number of factors, for example, whether the target enantiomer is more or less retained, physicochemical parameters, and cost factors. As a consequence, an effective strategy has to be implemented to master such complexity and provide results that have some general validity, that is, beyond the specific case considered. Here we define such a strategy, whereas in the next parts of this section we will provide the results of the analysis.
The first step is that of highlighting-for the first time in the literature-the role of the rate of the racemization reaction, and of its relative conversion in determining for a specific set of system parameters whether IP is worth considering and, in this case, what the optimal relative conversion is. This is discussed in section 4.3.

The second step, reported in section 4.4, will elucidate how such a conclusion depends on the values of three key technical parameters characterizing the system, namely the racemization rate constant, $k_{\mathrm{r}}^{\prime}$, the selectivity of the chiral stationary phase, $a$, and the characteristic upstream residence time, $\tau_{\text {up }}$, which lumps the effects of the technical challenges associated with the upstream section of the process.

The final step addresses economical considerations, by analyzing the effect of costs on the techno-economic performance of the two processes considered. As reported in section 4.5 , we will consider the effect of variations of the costs of the three most important sections of the process, namely the upstream section, the chromatographic separation, and the racemization section, on determining which process is preferable.

The parameter values used in this simulation study are based on the experimental characterization reported in the first part of this series. ${ }^{10}$ These values are then varied to elucidate the effects summarized above (see Table 2: constant parameters in the upper part; parameters varied in the scope of the parametric analysis in the lower part, with the reference values in bold).

To be general, the comparative assessment is carried out for systems subjected to more than one nonlinear adsorption isotherm, namely to the four generalized Langmuir isotherms, where the adsorbed phase concentration, $n_{i}$, is defined as

$$
n_{i}=\frac{H_{i} c_{i}}{1+p_{1} K_{1} c_{1}+p_{2} K_{2} c_{2}} \quad,(i=1,2)
$$

where $p_{1}= \pm 1$ and $p_{2}= \pm 1$. When $p_{1}=p_{2}=1$, the equation corresponds to the classical binary Langmuir isotherm $(\mathbf{L})$, while when both parameters are equal to -1 , the antiLangmuir isotherm (A) applies. The mixed cases are also possible: the type-1 mixed Langmuir isotherm $\left(\mathbf{M}_{\mathbf{1}}\right)$, where $p_{1}$ $=1=-p_{2}$, and the less and more retained component follow a Langmuir and anti-Langmuir isotherm, respectively; this is the isotherm characterizing the experimental system studied in part 1 of this series. ${ }^{10}$ The opposite case defines the type- 2 mixed Langmuir isotherm $\left(\mathbf{M}_{2}\right)$, where $p_{1}=-1=-p_{2}$.

In all cases analyzed in the following, where a generalized Langmuir isotherm has been considered, the parameter $x$ defined in section 3.2 is set to zero. The reason is 2 -fold. On the one hand, recycling more of the target enantiomer appears intuitively to be suboptimal. On the other hand, when $x \neq 0$ there are no exact criteria to determine optimal values of the flow rate ratios $m_{j}$ for nonlinear isotherms. Such criteria exist however for systems subjected to linear isotherms, and therefore we report the results for $x \neq 0$ in the case of linear isotherms in section 5.2. Additionally, we compare these results with those of a full simulation study for one case of a selected type of nonlinear isotherm.

4.2. Technical Issue Related to the SMB Feed Compositions. The so-called triangle theory approach to the design of SMB separations for systems subjected to the generalized Langmuir isotherm is reported in detail in previous publications of one of the authors (M.M.). ${ }^{19,20}$ There, it is 
Table 2. Input Parameters of the SMB and Integrated Process Model: Upper Part with the Constant Parameters, and Bottom Part with Those Varied for Investigation (The Reference Values Are Reported in Bold and the Range Examined in Brackets)

\begin{tabular}{|c|c|c|c|}
\hline variable & unit & value & description \\
\hline$N_{\min }$ & & 1000 & minimum number of stages \\
\hline$h$ & $\min$ & {$[0.0022,0.0028]$} & column efficiency constant ${ }^{a}$ \\
\hline$\Delta P_{\max }$ & bar & 40 & maximum pressure drop \\
\hline$\lambda$ & bar $\min \mathrm{cm}^{-2}$ & 0.03 & pressure drop factor \\
\hline$\epsilon^{*}$ & & 0.72 & SMB column void fraction \\
\hline$H_{1}$ & & 0.95 & Henry's constant of less retained component (1) \\
\hline $\mathrm{H}_{2}$ & & varies with $a$ & Henry's constant of more retained component (2) \\
\hline$K_{1}$ & $\mathrm{~L} \mathrm{~g}^{-1}$ & 0.03 & equilibrium constant of component 1 \\
\hline$K_{2}$ & $\mathrm{~L} \mathrm{~g}^{-1}$ & 0.31 & equilibrium constant of component 2 \\
\hline$k_{\mathrm{s}, 2} a_{\mathrm{v}}$ & $\mathrm{s}^{-1}$ & 5.25 & to determine the mass transfer coefficient ${ }^{b}$ \\
\hline$k_{\mathrm{s}, 1} a_{\mathrm{v}}$ & $\mathrm{s}^{-1}$ & 4.1 & \\
\hline$D_{\mathrm{L}, \mathrm{i}} \epsilon_{\mathrm{b}} / u_{0}$ & $\mathrm{~m}$ & $2.55 \times 10^{-5}$ & to determine the dispersion coefficient ${ }^{c}$ \\
\hline$\epsilon_{\mathrm{r}}$ & & 0.409 & reactor bed void fraction \\
\hline$\tau_{\mathrm{v}}$ & $\min$ & 10 & evaporator residence time \\
\hline$q_{0}$ & $g \min ^{-1}$ & 1 & total amount of enantiomer $i$ in overall feed \\
\hline$c^{*}$ & $\mathrm{~g} \mathrm{~L}^{-1}$ & 8.1 & Solubility of pure enantiomer $i$ at $22{ }^{\circ} \mathrm{C}$ \\
\hline$k_{\mathrm{r}}^{\prime}$ & $\min ^{-1}$ & $\mathbf{0 . 0 3 5},[0,0.1]$ & racemization rate constant \\
\hline$a$ & & $3.3,[1,4]$ & selectivity, $H_{2} / H_{1}$ \\
\hline$\tau_{\text {up }}$ & d & $0.5,1,2,4$ & upstream process residence time \\
\hline$x$ & & $\mathbf{0},[0, \infty)$ & target enantiomer in distomer stream ${ }^{d}$ \\
\hline$y$ & & $(0, \infty)$ & $2 / \xi-2+x$, with $\xi=(0,1)$ \\
\hline
\end{tabular}

${ }^{a}$ Value obtained from a simplified van Deemter equation ${ }^{30}$ as

$$
h=\frac{2}{\left(1-\epsilon^{*}\right) H_{2} k_{s, 2} a_{\mathrm{v}}}\left(\frac{\left(1-\epsilon^{*}\right) H_{2}}{\epsilon^{*}+\left(1-\epsilon^{*}\right) H_{2}}\right)^{2}
$$

with the transport parameters obtained in part 1 of this series. ${ }^{10}{ }^{b}$ Where $k_{\mathrm{s}, i}$ is the mass transfer coefficient and $a_{\mathrm{v}}$ the specific surface. ${ }^{c} \mathrm{Where} D_{\mathrm{L}, i}$ is the dispersion coefficient, $\epsilon_{\mathrm{b}}$, and $u_{0}$ the superficial velocity. ${ }^{d}$ Varied in the linear isotherm case only.

stated that the theory applies only in the case of feed compositions, for which the denominator of the adsorption isotherm (eq 22) is positive, that is, where $1+p_{1} K_{1} c_{1}^{\mathrm{F}}+p_{2} K_{2} c_{2}^{\mathrm{F}}$ $>0$, with $c_{1}^{\mathrm{F}}$ and $c_{2}^{\mathrm{F}}$ being the feed concentrations of the two species to be separated. The boundaries of the complete separation region and the coordinates of the optimal point, indicated as point $\mathbf{w}$ in the previous works, are given in terms either of the feed concentrations, or of the two parameters $\omega_{1}^{\mathrm{F}}$ and $\omega_{2}^{\mathrm{F}}$, which can be calculated from the feed composition and fulfill certain useful properties. ${ }^{19}$ The constraint related to the denominator of the adsorption isotherm is relevant only for the $\mathbf{M}_{1}$ and $\mathbf{A}$ isotherm, since the denominator consists only of positive terms in the $\mathbf{L}$ isotherm, while in the $\mathbf{M}_{2}$ isotherm another constraint prevails (eq 7 in Mazzotti ${ }^{19}$ ). In the course of this work, and thanks to a real element of serendipity, we have come to realize that such limitation of scope is unnecessary.

Let us focus then on the two isotherms of interest, and notice that there are two constraints on the SMB feed concentration and on the concentrations in the SMB in general, namely the requirements that the solubility cannot be overcome (see section 3.3) and that the isotherm denominator be positive. The former has a physical underpinning. The latter on the contrary would be relevant only under the assumption that the adsorption isotherm has a very broad range of applicability, which obviously might not always be the case. The following discussion is justified by the assumption that both requirements are fulfilled, and is heavily based on technical details of the derivations in the earlier paper $^{19}$ (it is worth signaling a typo there, whereby in eq $39 m_{4}$ should replace $m_{3}$ ). Since for the sake of brevity it is impossible to report the whole argument in a self-contained manner, the interested (and patient) readers are recommended to read what follows with the mentioned reference ${ }^{19}$ in front of them. Those, who are not interested in the details, can move to the next section while just retaining the key conclusion: that "in the case of the type-1 mixed Langmuir isotherm $\mathbf{M}_{1}$ and of the anti-Langmuir isotherm $\mathbf{A}$ and for complete separation operating conditions, even when the SMB feed composition violates the constraint on the denominator of the adsorption isotherm, all compositions within the SMB unit comply with such constraint".

The ultimate physical reason is that the SMB feed is a solution that as such never sees the adsorbent. Before contacting the stationary phase in the SMB columns the feed gets mixed and diluted with the outlet fluid stream from section 2. The composition of the inlet fluid stream to section 3 depends on the relative amounts of the feed and of the section 2 outlet, which in turn are constrained by the requirement of complete separation. It is intuitive that the higher is the total feed concentration, the smaller is the feed flow rate, and hence the larger is the dilution effect. As a matter of fact, whatever the feed composition, the feed is always diluted enough so that the composition of the inlet stream to section 3 yields a positive denominator in the relevant expression of the adsorption isotherm.

From a mathematical viewpoint, this conclusion can be proven by considering eqs 47 and 48 of the earlier paper. ${ }^{19}$ They are two quadratic equations, the solutions of which are the $\omega$ values characterizing the composition states prevailing in 
sections 2 and 3 of the SMB under complete separation conditions (see Figure 2 of the same paper ${ }^{19}$ ). The coefficients of these two equations, given by eqs 49 to 52 , depend on the feed concentrations $c_{1}^{\mathrm{F}}$ and $c_{2}^{\mathrm{F}}$ directly, not through the values of $\omega_{1}^{\mathrm{F}}$ and $\omega_{2}^{\mathrm{F}}$. Sections 4.2.1 and 4.2.3 of the earlier paper ${ }^{19}$ prove that the solutions of the two quadratic equations fulfill always the constraints on the $\omega$ values that guarantee that the corresponding composition state yields a positive denominator, whatever the values of $c_{1}^{\mathrm{F}}$ and $c_{2}^{\mathrm{F}}$. This proves the aforementioned key conclusion.

On the basis of this observation, in the case of the mixed Langmuir isotherm $\mathrm{M}_{1}$, it is possible to write the coordinates of the optimal point (vertex of the triangle-shaped complete separation region, $\mathbf{w}_{\mathbf{M} 1}$ ) in terms of the feed concentrations (in Table 4 of the earlier paper ${ }^{19}$ such coordinates are given in terms of $\omega_{1}^{\mathrm{F}}$ and $\omega_{2}^{\mathrm{F}}$, which is correct only for feed concentrations not violating the constraint on the isotherm denominator). Such coordinates are determined by intersecting lines aw and bw, the equations in terms of $c_{1}^{\mathrm{F}}$ and $c_{2}^{\mathrm{F}}$ of which are reported in Table 3 of the previous paper, ${ }^{19}$ thus obtaining

$$
\begin{aligned}
& m_{2}^{\mathbf{w}_{\mathrm{M}_{1}}}=H_{1}\left\{1+\frac{\left(H_{2}-H_{1}\right) K_{2} c_{2}^{\mathrm{F}}}{H_{2}-H_{1}+H_{2} K_{1} c_{1}^{\mathrm{F}}+H_{1} K_{2} c_{2}^{\mathrm{F}}}\right\} \\
& m_{3}^{\mathbf{w}_{\mathrm{M}_{1}}}=H_{2}\left\{1-\frac{\left(H_{2}-H_{1}\right) K_{1} c_{1}^{\mathrm{F}}}{H_{2}-H_{1}+H_{2} K_{1} c_{1}^{\mathrm{F}}+H_{1} K_{2} c_{2}^{\mathrm{F}}}\right\}
\end{aligned}
$$

In the case of the anti-Langmuir isotherm it can be shown (using eq 88 of the earlier paper ${ }^{19}$ ) that the value of $\omega_{1}^{\mathrm{F}}$ fulfills its constraints; that is, $H_{1} \leq \omega_{1}^{\mathrm{F}} \leq H_{2}$ (eq 10 of the reference above $^{19}$ ) whatever the feed composition. Therefore, the coordinates of the vertex of the complete separation region $\mathbf{w}_{\mathrm{A}}$ can be calculated in terms of the feed concentrations and of $\omega_{1}^{\mathrm{F}}$, that is, without using $\omega_{2}^{\mathrm{F}}$. This requires that the equation of the line sw, given in Table 3 of the earlier paper, ${ }^{19}$ be modified algebraically by using eqs 13 and 14 of the same paper, thus obtaining

$$
m_{2} \frac{K_{1} c_{1}^{F}}{\omega_{1}^{F}-H_{1}}+m_{3} \frac{K_{2} c_{2}^{F}}{\omega_{1}^{F}-H_{2}}=1
$$

Intersecting line sw given by this equation and line aw from Table 3 of the earlier paper ${ }^{19}$ yields the following coordinates of the vertex of the complete separation region, which applies whatever the feed composition:

$$
\begin{aligned}
& m_{2}^{\mathbf{w}_{\mathrm{A}}}=\frac{\omega_{1}^{\mathrm{F}}-H_{1}}{K_{1} c_{1}^{\mathrm{F}}}\left\{1+\frac{H_{2} K_{2} c_{2}^{\mathrm{F}} \omega_{1}^{\mathrm{F}}}{H_{1}\left(H_{2}-\omega_{1}^{\mathrm{F}}\right)}\right\} \\
& m_{3}^{\mathbf{w}_{\mathrm{A}}}=\frac{\omega_{1}^{\mathrm{F}} H_{2}}{H_{1}}
\end{aligned}
$$

It is worth noting that some of the SMB feed compositions used in the experimental runs reported in the first part of this series, ${ }^{10}$ where the mixed Langmuir isotherm $\mathbf{M}_{\mathbf{1}}$ applies, fall in the category discussed in this section, as it can be easily verified. This is in fact the real element of serendipity mentioned at the beginning of this section, namely that we ran those experiments without realizing that in some cases the feed composition selected would yield a negative denominator in the expression for the adsorption isotherm. When we realized that, we started the reflections that led to the conclusions sketched in this section.

4.3. Determining Optimal Racemization Conditions. The effect of changing the relative conversion of the racemization reaction on the productivity of the IP is illustrated in Figure 3. The three curves are obtained for the

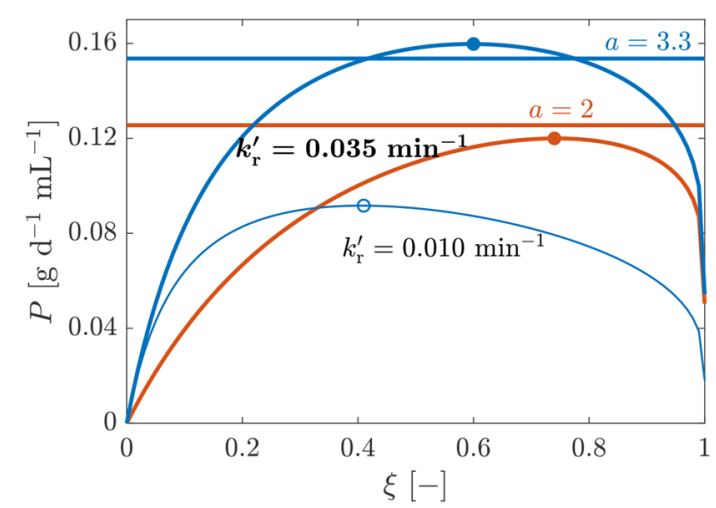

Figure 3. Productivity of SP (horizontal lines) and IP (curves) as a function of extent of reaction for different values of the racemization rate constant, $k_{\mathrm{r}}^{\prime}$, and selectivity, $a$. The circle indicates the maximum $P_{\mathrm{IP}}$, corresponding to the optimal conversion, $\xi_{\text {opt }}$. The case of $\mathbf{M}_{\mathbf{1}}$ isotherm, $Q_{T}=Q_{R}$.

set of reference system parameters, and for three combinations of kinetic rate constant, $k_{\mathrm{r}}^{\prime}$ (which equals 0.010 and 0.035 $\min ^{-1}$ for thin and thick lines, respectively), and selectivity, $a$ (which equals 2.0 and 3.3. for the red and the blue lines, respectively).

One readily observes that productivity of the IP approaches zero in two cases. First, it does so for $\xi \rightarrow 0$, because $y \rightarrow \infty$ due to eq 4 and SMB feed flow rate and volume approach infinity due to eqs 5 and 14 . Then, productivity approaches zero also for $\xi \rightarrow 1$, because both residence time, $\tau_{\mathrm{r}}$, and volume, $V_{\mathrm{r}}$, of the racemization reactor become infinitely large due to eqs 18 and 19. It is also noteworthy, though intuitive, that increasing either $k_{\mathrm{r}}^{\prime}$ or $a$ while keeping the other parameter constant improves productivity over the whole range of $\xi$ values, particularly at the maximum of the curve, that is defined as the optimal relative conversion, $\xi_{\text {opt }}$ for a given set of system parameters.

The horizontal straight lines in Figure 3 show the productivity of the SP for the same system parameters, which is obviously independent of the racemization reaction. It can be readily observed that there are combinations of the two parameters where the optimal performance of the IP is better than that of the SP (thick blue lines, for the larger values of $k_{\mathrm{r}}^{\prime}$ and $a$ ). In other cases the SP performs always better than the IP, and this can happen when either of the two parameters becomes smaller. In the following, whenever we compare the IP and SP performances, we refer to the optimal IP performance attained at $\xi_{\text {opt }}$.

With reference to the discussion in section 3.3 about which SMB stream exhibits the highest concentration, we illustrate in Figure 4 some typical behaviors. The three subfigures refer to different isotherms, namely type- 1 mixed, Langmuir and antiLangmuir; there we plot the reduced concentration of one or the other enantiomer, that is, the quantity $S_{\mathrm{i}}=c_{\mathrm{i}} / c^{*}$, in the feed (solid line), extract (dotted line), and raffinate (dashed line), as a function of the selectivity of the chiral stationary phase. In 


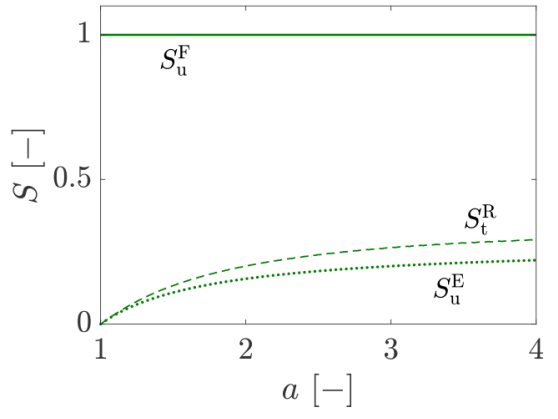

(a)

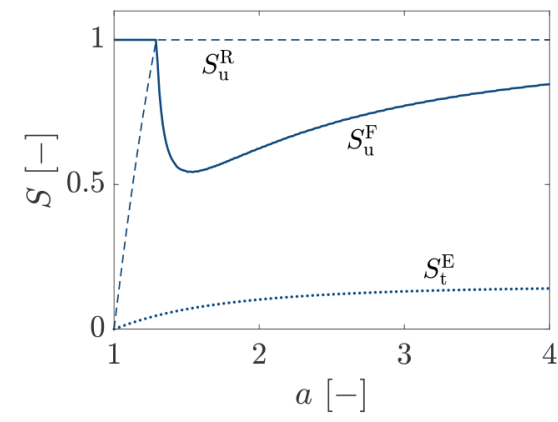

(b)

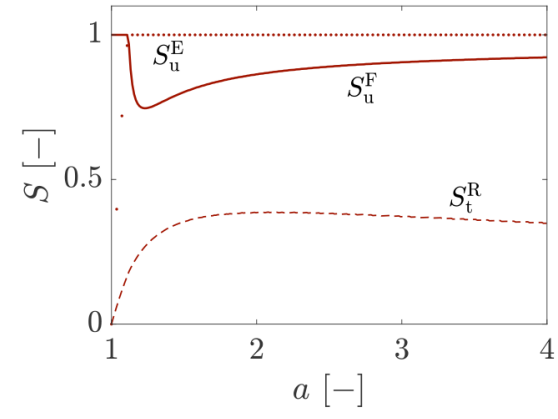

(c)

Figure 4. Effect of the selectivity, $a$, on the concentration of the SMB streams in the IP at $k_{\mathrm{r}}^{\prime}=0.035 \mathrm{~min}^{-1}$, indicated as supersaturation values, $S_{i}$, at the corresponding $\xi_{\text {opt }}$ of the relevant components and streams in different cases analyzed: (a) $M_{1}$ isotherm, $Q_{T}=Q_{R}(b) L$ isotherm, $Q_{T}=Q_{E}$, and (c) A isotherm, $Q_{T}=Q_{R}$.

the first and in the last case, the maximum concentration is always that in the feed and in the extract, respectively. In the second case, the feed is limiting up to a threshold $a$ value, beyond which the raffinate concentration is controlling; the feed distomer concentration is reduced by the procedure defined in section 3.3 accordingly, as illustrated in Figure $4 \mathrm{~b}$. The reason for the different behaviors exhibited by the different isotherms stems from the shape of the complete separation region in the operating parameter space, and from how the optimal set of $m_{j}$ values changes for changing feed composition (the reader is referred to earlier literature ${ }^{19,20}$ where these effects are illustrated).

It is worth noting that there are cases in which the IP flow sheet can be simplified. When the limiting concentration is that of the distomer in the stream with flow rate $Q_{U}$, then the concentration at the reactor inlet, $c_{\mathrm{w}}^{\mathrm{r}}$ is already at the desired value. Therefore, there is no need to further concentrate the solution, and the evaporator on that SMB outlet stream can be eliminated. This situation may occur in the case of the Langmuir isotherm when the eutomer is collected in the extract, as shown in Figure $4 b$, for $a>1.26$; similarly, in the case of the $\mathbf{A}$ isotherm (Figure $4 \mathrm{c}$ ), when the concentration of the distomer in the extract, $S_{\mathrm{u}}^{\mathrm{E}}$, is the limiting one and the eutomer is collected in the raffinate. On the other hand, in the $\mathbf{M}_{1}$ case illustrated in Figure $4 \mathrm{a}$ both outlet concentrations are well below the upper limit, thus making two evaporating units necessary for all values of $a$. The possible scenarios for the different isotherms considered are reported in Table 3, while other simulation results are presented in the Supporting Information.

4.4. Technical Comparative Assessment. The behavior illustrated in Figure 3 indicates that in the system parameters space there must be a threshold locus of points that separates

Table 3. Limiting Concentration of the Process and the Corresponding Number of Evaporating Units Needed for Each of the Four Isotherm Cases, When the Eutomer Is Collected in Either the Raffinate or the Extract Stream

$\begin{array}{ccc} & \text { product in raffinate } & \text { product in extract } \\ \text { isotherm } & Q_{T}=Q_{R} & Q_{T}=Q_{E} \\ \mathbf{L} & c_{\mathrm{t}}^{\mathrm{R}}, 2 \text { evp. } & c_{\mathrm{u}}^{\mathrm{R}}, 1 \text { or } 2 \text { evp. } \\ \mathbf{A} & c_{\mathrm{u}}^{\mathrm{E}}, 1 \text { evp. } & c_{\mathrm{t}}^{\mathrm{E}}, 2 \text { evp. } \\ \mathbf{M}_{\mathbf{1}} & c_{\mathrm{u}}^{\mathrm{F}}, 2 \text { evp. } & c_{\mathrm{u}}^{\mathrm{F}}, 2 \text { evp. } \\ \mathbf{M}_{2} & c_{\mathrm{u}}^{\mathrm{E}}, 1 \text { evp. } & c_{\mathrm{t}}^{\mathrm{E}}, 2 \text { evp. }\end{array}$

the regions where the SP and the IP prevail. Such space is three-dimensional in our analysis, because the racemization rate constant, $k_{\mathrm{r}}^{\prime}$, the selectivity of the chiral stationary phase, $a$, and the residence time in the upstream section of the process, $\tau_{\text {up }}$, have been identified as the key parameters controlling the relative performance of the two processes.

The threshold surface thus ensuing is visualized through its 2-D intersections with horizontal planes for a fixed value of $\tau_{\text {up }}$ which are plotted as projections on the plane having as coordinates $k_{\mathrm{r}}^{\prime}$ and $a$. These intersection lines are visible in Figure 5 as an interface separating a white region on the lefthand side, where the SP prevails, from a colored region on the right-hand side, where the IP exhibits higher performance. The four subfigures apply to the four generalized Langmuir isotherms for the case where the eutomer is less retained; hence, it is collected in the raffinate (similar figures for the case where the eutomer is more retained and collected in the extract are provided in the Supporting Information). Note that all weights accounting for the costs of the different process units that appear in the denominator of eq 21 have been kept constant, so as to focus on the role of the technical features of the process. How changing $\tau_{\text {up }}$ affects the position of the threshold line is illustrated in Figure 6, again for the eutomer in the raffinate (the case of the eutomer in the extract is reported in the Supporting Information).

Let us analyze one of the subfigures of Figure 5 more in detail, for example, Figure 5c. The thicker black contour lines are iso-productivity lines, whereas the thinner gray contour curves within the colored region are iso- $\xi_{\text {opt }}$ lines. Obviously, productivity contour lines in the white region where the SP prevails are parallel to the $k_{\mathrm{r}}^{\prime}$-axis because there is no racemization in the SP. The three pairs of parameters used to draw the curves in Figure 3 are the coordinates of the three symbols (circles) also plotted in the figure.

There are a few remarks worth making based on the analysis of Figures 5 and 6.

First, as one might expect, systems characterized by different generalized Langmuir adsorption isotherms behave in a qualitatively very similar manner. Because of the broad range of types of interaction between the two enantiomers described by the four isotherms considered here, this observation confirms that the trends observed in this analysis are general, whatever the adsorption isotherm.

Second, also as expected, the SP is to be preferred when the racemization reaction is rather slow and when the chiral 


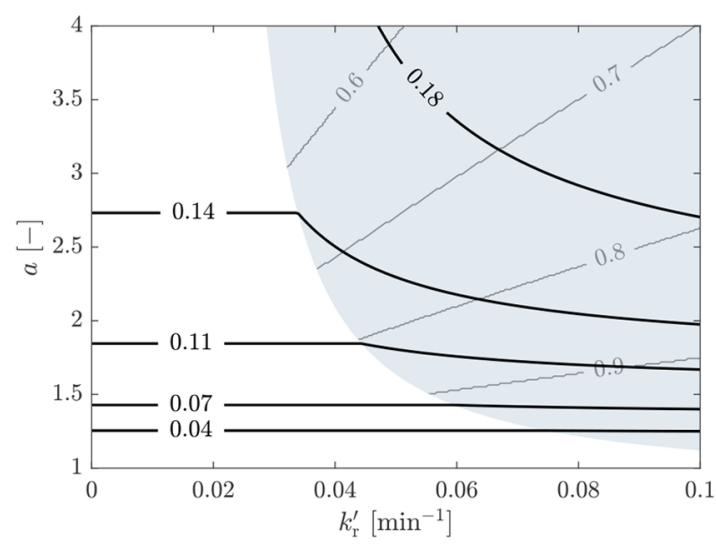

(a)

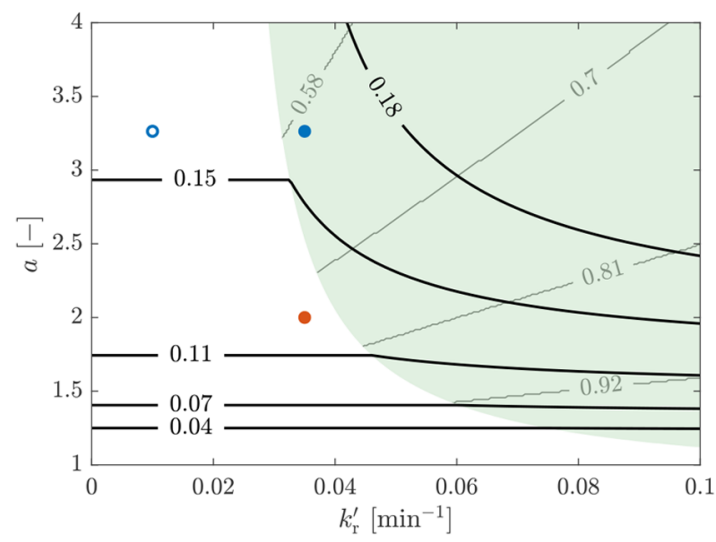

(c)

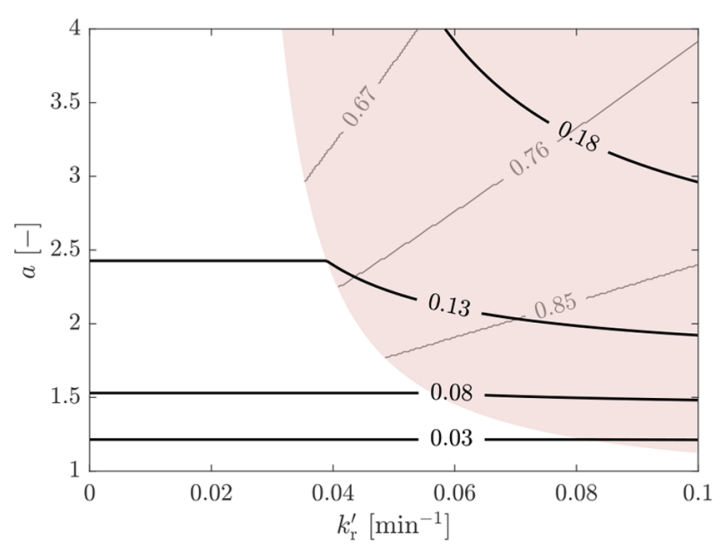

(b)

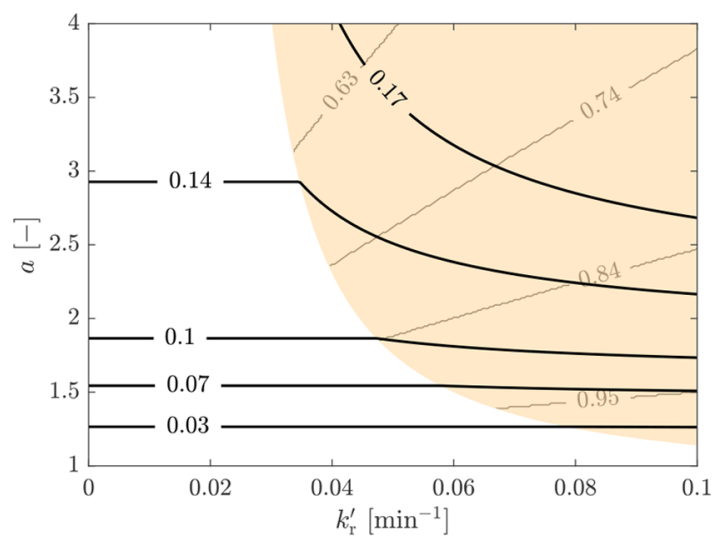

(d)

Figure 5. Effect of the selectivity, $a$, and racemization rate, $k_{\mathrm{r}}^{\prime}$, on the productivity (in $\mathrm{g} \mathrm{d}^{-1} \mathrm{~mL}^{-1}$, black contour lines) of either SP (white area) or IP (colored area). For IP the maximum value of $P_{\text {IP }}$ is reported with the corresponding $\xi_{\text {opt }}$ (gray contour lines): (a) Langmuir, (b) anti-Langmuir, (c) type-1 mixed Langmuir, and (d) type-2 mixed Langmuir isotherm. The markers in panel c report the cases analyzed in Figure $3\left(\tau_{\text {up }}=1 \mathrm{~d}, Q_{\mathrm{T}}=\right.$ $\left.Q_{R}\right)$.

stationary phase offers only low selectivity. To cross into the region where the IP outperforms the SP, one of two things must happen, or both: either the SMB separation becomes easier at high $a$ (in a region corresponding to low levels of optimal racemization conversion), or the racemization reaction speeds up (ending up in a region where $\xi_{\text {opt }}$ is higher). Therefore, one can conclude that if one phenomenon is unfavorable, for example, racemization rate too low, the IP performs better than the SP only if the selectivity is high enough, thus compensating for the inefficiency of the reaction.

Finally, increasing the upstream residence time, that is, increasing the volume of the upstream section, reduces significantly the size of the region of the parameter space where the SP is attractive as shown for the eutomer in the raffinate case in Figure 6. This is expected, since for a given throughput the SP requires an amount of feed equal to $(2(2+$ $x) q_{0}$ ), which is at least twice of that needed by the IP, that is, $2 q_{0}$.

4.5. Factoring in the Uncertainty in Costs. The sensitivity of the results on the costs has been investigated by varying the unitary costs and the corresponding weights of $\mathrm{SMB}$, racemization reactor and upstream section of the process, that is, $\pi_{\mathrm{SMB}}, \pi_{\mathrm{r}}$, and $\pi_{\mathrm{up}}$ in eq 21 , while keeping $\pi_{\mathrm{v}}$ constant. The outcome is illustrated in Figure 7 panels $a$ and $b$ for one specific system characterized by the type-1 mixed Langmuir isotherm; results for the other isotherms are similar and not worth reporting here in the interest of brevity. The two figures are drawn following the same graphical conventions applied to draw Figures 5 and 6.

The key messages from these two diagrams are as follows. First, increasing the costs of racemization and chromatographic separation has a similar effect as reducing the racemization rate constant and the chromatographic selectivity, namely, it favors the SP with respect to the IP. Second, decreasing the cost of the upstream section has the same effect of decreasing the average residence time $\tau_{\text {up }}$, namely it makes the SP more favorable than the IP. These two observations are general and intuitive. Another noteworthy remark is that the projections of the threshold surface on the plane with coordinates $\pi_{\mathrm{SMB}}$ and $\pi_{\mathrm{r}}$ are very steep, thus indicating a bigger role of the cost of the racemization reactor than of that of the SMB.

\section{EFFECT OF ADDITIONAL DESIGN PARAMETERS}

The analysis in the previous section, the results of which are illustrated in Figures 5, 6, and 7, has been carried out in the scope of the equilibrium theory of nonlinear chromatography, more specifically of the so-called triangle theory. Equilibrium theory is extremely powerful in providing insight in the key phenomena determining the possibly complex behavior of nonlinear chromatography. Its application to SMB design is also extremely powerful in easily identifying regions with different separation performances, particularly the region of 


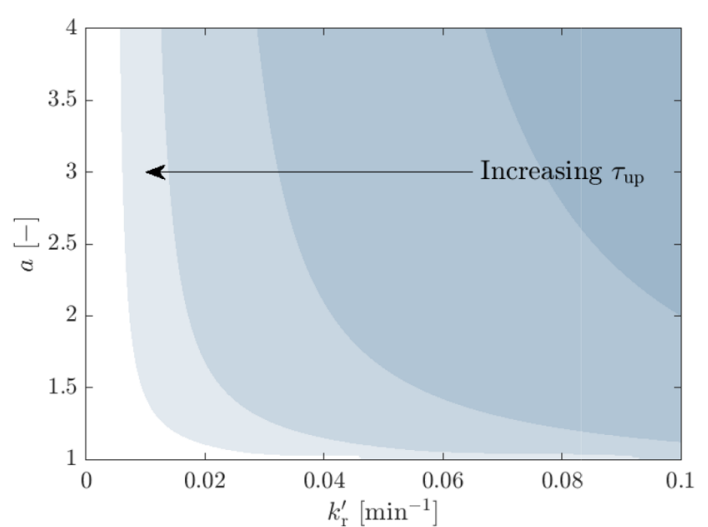

(a)

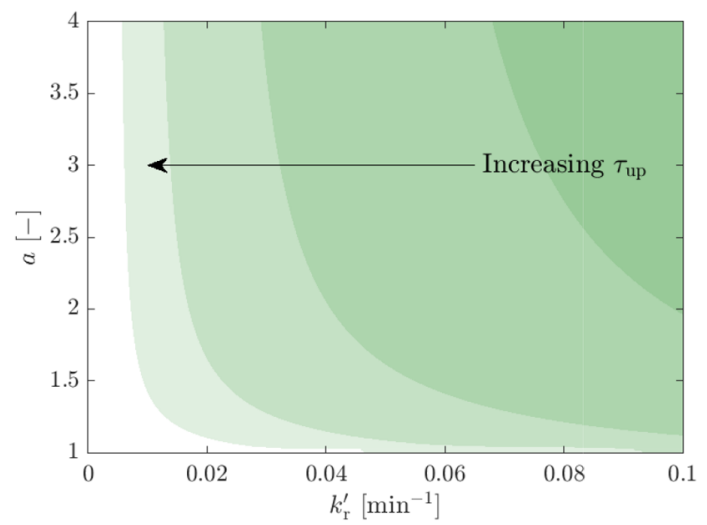

(c)

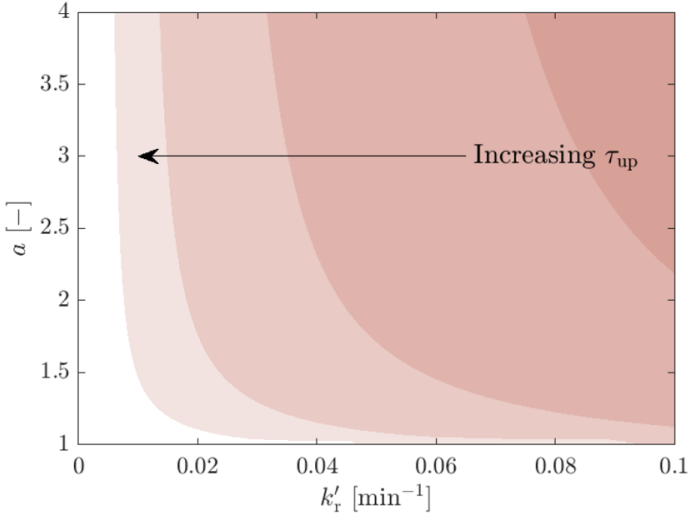

(b)

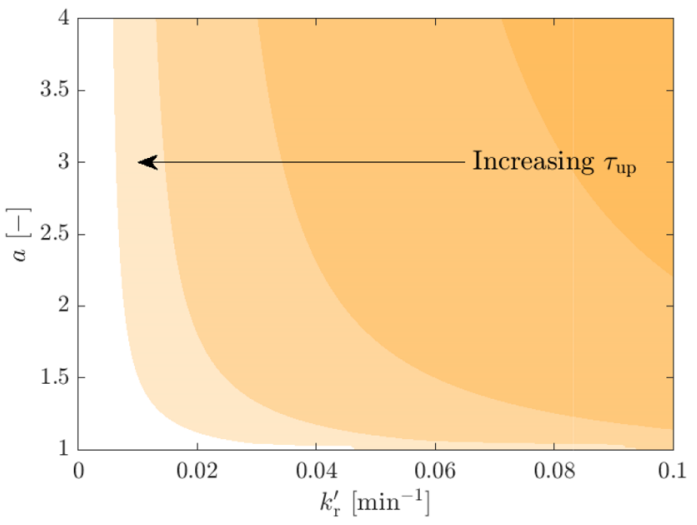

(d)

Figure 6. Effect of the upstream residence time, $\tau_{\mathrm{up}}=0.5,1,2,4 \mathrm{~d}$, on the productivity of SP or IP as a function of selectivity, $a$, and racemization rate, $k_{\mathrm{r}}^{\prime}$. White (colored) area for conditions at which the $P_{\mathrm{SP}}\left(P_{\mathrm{IP}}\right)$ is higher: (a) Langmuir, (b) anti-Langmuir, (c) type-1 mixed Langmuir, and (d) type-2 mixed Langmuir isotherm. .

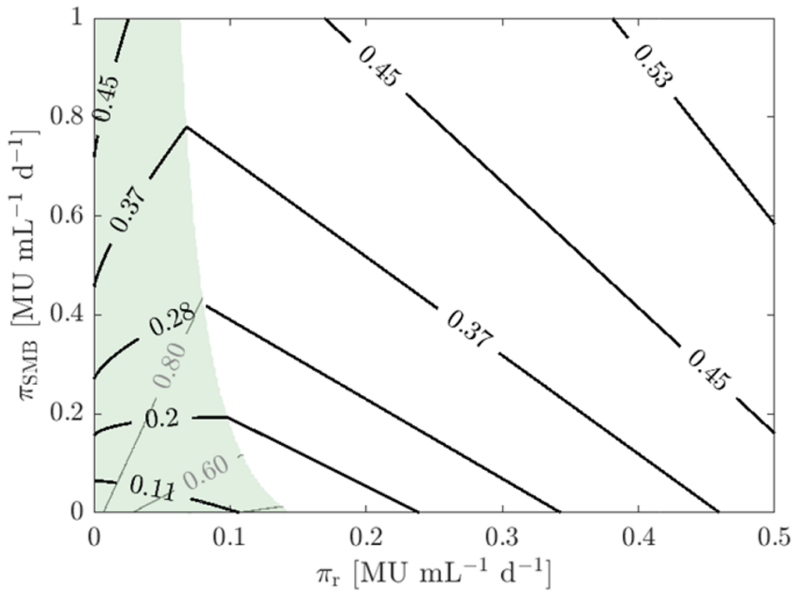

(a)

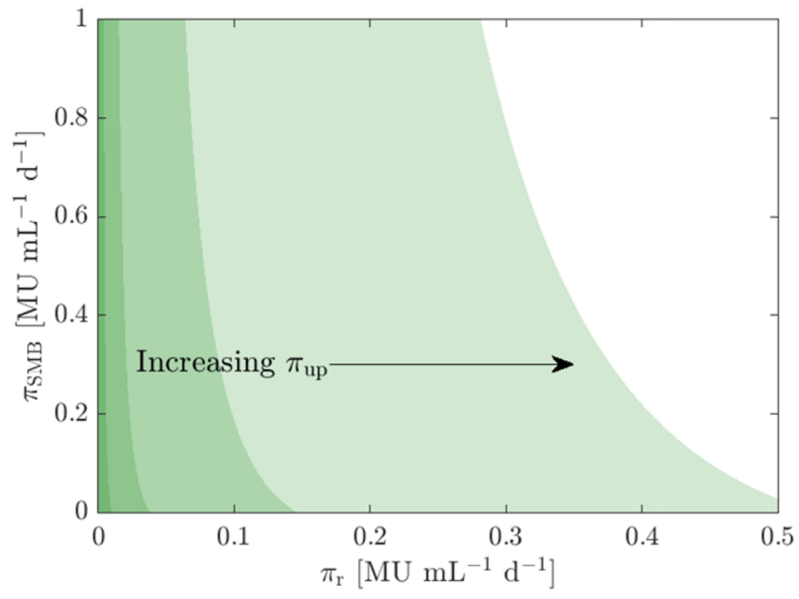

(b)

Figure 7. Effect of the cost factor on the productivity of SP (white area) or IP (green area) as a function of reactor, $\pi_{\mathrm{r}}$, and SMB, $\pi_{\mathrm{SMB}}$, cost parameters, for the $\mathbf{M}_{\mathbf{1}}$ isotherm. White (green) area for conditions at which the $P_{\mathrm{SP}}\left(P_{\mathrm{IP}}\right)$ performs best. (a) At constant $\tau_{\mathrm{up}}=1 \mathrm{~d}$ with black contour lines indicating the productivity value, and the gray ones indicating the corresponding $\xi_{\text {opt }}$ for the IP. (b) The effect of the feed costs on the productivity comparison, $\pi_{\mathrm{up}}=0.001,0.03,0.01,0.03\left(\mathrm{M}_{1}\right.$ isotherm, $\left.Q_{\mathrm{T}}=Q_{\mathrm{R}}\right)$.

complete separation, and in predicting and explaining the effect of changing the key operating parameters on separation performance. It is the ideal tool to perform the type of comparative analysis carried out in this work, because it combines simplicity (from a computational perspective) and thoroughness (from the perspective of the completeness with which it accounts for most key effects in nonlinear SMB chromatography). 
However, triangle theory applied to nonlinear adsorption isotherms has two important limitations: it does not help (i) in determining the size of the SMB columns (see section 5.1), and (ii) in selecting specific values of the flow rate ratios $m_{j}$ that lead to a given incomplete separation performance (which in the context of this paper implies specifying a value $x>0$ ) (see section 5.2). Nevertheless, in the analysis above, the size of the SMB unit is key in enabling the comparison of the productivity of different process configurations (see section 3.3). Hence, it has been determined by constraining the maximum pressure drop and the minimum number of theoretical stages, $N_{\min }$, that is, by enforcing conditions that do not belong to the equilibrium theory framework.

5.1. Minimum Number of Theoretical Stages. In the scope of equilibrium theory, as we use it here, the chosen value of $N_{\text {min }}=1000$ is assumed to be large enough to guarantee that the desired high purity of both product streams is indeed attained when choosing operating conditions within the complete separation region determined by the triangle theory. In other words, this is a statement about column efficiency that ultimately contributes to the determination of the SMB size. The size of the SMB unit, in terms of $V$ and $L$, and the switch time increase with increasing $N_{\min }$ and constant $m_{j}$ values, and vice versa, according to eqs 14,15 , and 16 . We expect that the effect of changing $N_{\text {min }}$ would be qualitatively the same for both processes and all isotherms considered and all cases analyzed in this work.

To evaluate the effect of the choice of this design parameter on the IP and SP operations, we have run simulations for the case of a type-1 mixed Langmuir isotherm with $N_{\min }=100$ and $x=0$. With reference to the plane of coordinates $k_{\mathrm{r}}^{\prime}$ and $a$ in Figure 8 , the new results are compared to those already

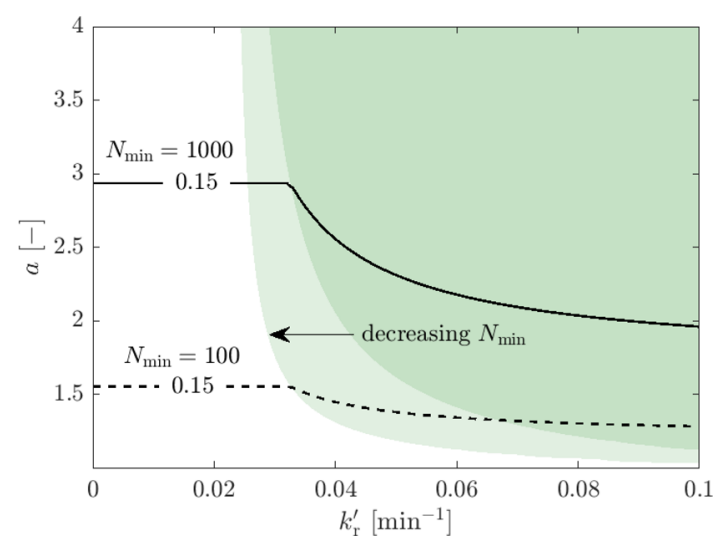

Figure 8. Effect of $N_{\text {min }}=1000,100$ on $P_{\mathrm{IP}}$ (green area) and $P_{\mathrm{SP}}$ (white area) as a function of selectivity $a$ and racemization rate $k_{r}^{\prime}$, for the case of the $\mathbf{M}_{1}$ isotherm with the product in the raffinate stream. An iso-productivity line at $P=0.15 \mathrm{~g} \mathrm{~d}^{-1} \mathrm{~mL}^{-1}$ is plotted for $N_{\min }=$ 1000 (solid black line) and $N_{\min }=100$ (dashed black line).

presented in section 4.4 (Figure $5 c, N_{\min }=1000$ ). The comparison of the iso-productivity line at $P=0.15 \mathrm{~g} \mathrm{~d}^{-1} \mathrm{~mL}^{-1}$ for the two $N_{\min }$ values indicates that by decreasing $N_{\min }$ the productivity of the SP and the IP increases, consistently with the effect of $N_{\min }$ on the SMB size. Moreover, we observe that the threshold line separating the region where the SP has better performance (white) and the region where the IP outperforms the SP (green) shifts, and that such a shift is relatively small. This shows that the reduction of the SMB volume similarly affects both processes. On the basis of these observations we conclude that the qualitative trends identified in this work are not strongly affected by the choice of $N_{\min }$, and therefore that the conclusions drawn on the relative productivity of both processes and its dependence on the system (see Figures 5 and 6) and cost (see Figure 7) parameters are general.

The analysis presented here allows us, for a specific system (with a given isotherm and given selectivity and kinetic rate constant), to identify the most favorable process, either IP or SP. Such selection should be followed by a detailed process optimization, where the optimal SMB operating conditions are determined following an approach similar to what is proposed in earlier literature. ${ }^{11,17,18,31-34}$ With that approach, the $N_{\min }$ constraint implemented here is relaxed, and also SMB operating conditions that correspond to rather small numbers of theoretical stages are considered. This is done under the separation requirement that the products' purity fulfills an assigned specification, and implies that the operating point in the $m_{j}$ space moves away from the vertex of the complete separation region, thus resulting in smaller values of $\left(m_{3}-\right.$ $m_{2}$ ), as shown by Kaspereit and collaborators. ${ }^{17,34}$

5.2. Incomplete Separation in the SMB. Previous contributions on conventional standalone SMB separation have demonstrated that relaxing purity specifications, that is, operating the SMB unit outside the complete separation region, leads to improvements in separation performance, particularly productivity. ${ }^{22,32,35,36}$ A performance improvement for lower purity requirements was observed also in the case of integrated processes, obtained by combining chromatography with either crystallization or racemization..$^{1,33,37}$ In the latter case, Fuereder et al. highlighted that reducing the purity requirements promotes better performance especially when the product is collected in the raffinate. ${ }^{12}$ In a recent paper, SeidelMorgenstern and co-workers specify the purity of the eutomer stream only, while the purity of the distomer stream to the racemization unit is exploited as a degree of freedom. ${ }^{16}$ This expands the operating window without adverse impact on the performance of the integrated process.

In light of these contributions, in the following, we discuss the effect of the SMB specifications, hence of choosing $x>0$, on the relative performance between the standalone SMB and the integrated process, when the effect of the racemization reaction conversion is also considered.

So far, we have enforced a complete separation constraint for both SP and IP, thus enabling the use of exact equations for the determination of optimal SMB operating conditions, that is, the $m_{j}$ values, in the case of all generalized Langmuir isotherms. We have not even speculated on a possible leak of the distomer into the eutomer stream, which is typically required to be at very high purity; such leakage would not make sense in the scope of the applications typically targeted by these processes. On the other hand, in our model the parameter $x$ (see Figures 1 and 2) accounts for the amount of desired enantiomer lost in the distomer stream; in the case of the SP such an amount would be a penalizing waste, whereas in the case of the IP it would be recycled again and again to the SMB unit, thus constituting a sort of ballast for the SMB process. By choosing $x>0$, the effect of such loss of target enantiomer on the performance of the IP and SP processes could be investigated following the same approach adopted for the case $x=0$, if we had relationships for the optimal $m_{j}$ values as a function of $x$. Such explicit relationships are however available only for linear isotherms, ${ }^{38}$ that is, where $n_{i}=H_{i} c_{i}(i=$ 


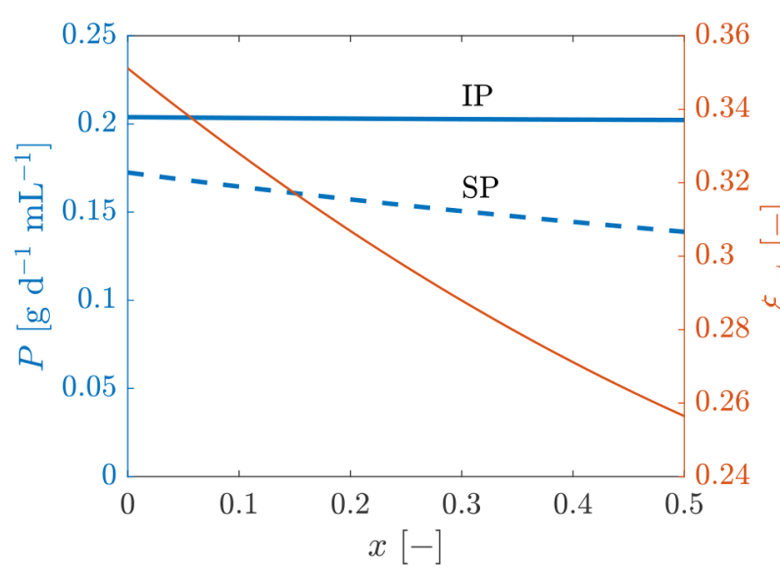

(a)

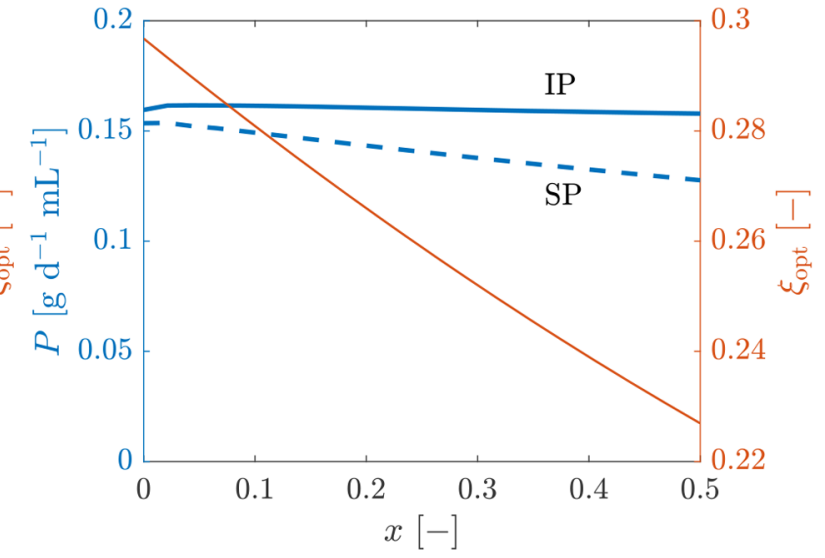

(b)

Figure 9. $P_{\mathrm{IP}}$ (full thick blue line) and $P_{\mathrm{SP}}$ (dashed blue line) as a function of $x$ when $Q_{\mathrm{T}}=Q_{\mathrm{R}}$ for (a) the linear isotherm case and (b) the $\mathbf{M}_{1}$ isotherm case. The corresponding $\xi_{\text {opt }}$ (thin red line) for the IP is plotted on the right axis. The simulation parameters are those reported in Table 2 for the reference case.

1, 2), with $H_{i}$ being the Henry's constant, and $H_{2}>H_{1}$. Therefore, we carry out a systematic investigation on the effect of incomplete separation in the case of linear isotherms, confident that conclusions about the behavior observed for linear isotherms can shed light on more general features, although linear isotherms are rather rare in preparative and SMB chromatography.

For a given pair of values $x$ and $y$, with $0 \leq x \leq y$, the purity in the stream where the distomer is collected equals $(2+y) /(2$ $+y+x)$ (see Figure 1). Using the results demonstrated by Rajendran, ${ }^{38}$ the $m_{2}$ and $m_{3}$ values associated to that purity level are as follows:

- if the eutomer is more retained and collected in the extract:

$$
\begin{aligned}
& m_{2}=H_{1} \\
& m_{3}=H_{2}+\frac{x\left(H_{2}-H_{1}\right)}{2}
\end{aligned}
$$

- if the eutomer is less retained and collected in the raffinate:

$$
\begin{aligned}
& m_{2}=H_{1}-\frac{x\left(H_{2}-H_{1}\right)}{2} \\
& m_{3}=H_{2}
\end{aligned}
$$

Note than in both cases $\left(m_{3}-m_{2}\right)=\left(H_{2}-H_{1}\right)(1+x / 2)$ and that $m_{1}=H_{2}$ and $m_{4}=H_{1}$ for linear systems. It is also worth noting that the value of $x$ is upper bounded by the constraint that all internal SMB flow rates must be positive. With the $m_{j}$ values above, the separation performances of the IP and the SP can be calculated and compared, as illustrated in Figure 9a. The general observation is that increasing $x$ has a negligible effect on the IP productivity, whereas it has a strong negative impact on the SP productivity. Moreover, the $\xi_{\text {opt }}$ decreases with increasing $x$. There are three reasons for that.

1. First, the size of the upstream section of the process does not change with $x$ in the IP case, whereas it scales with the overall amount fed to the SMB unit in the SP case, which is proportional to $2(2+x)$ and increases linearly with $x$.

2. Second, the size of the SMB unit does not change much with $x$. This is evident if one considers the SMB volume given by eq 14 , which is proportional to $Q_{F}$ and to the reciprocal of $\left(m_{3}-m_{2}\right)$. Both these quantities increase with $x$ because of the observation above and because of eq 5 .

3. Finally, we observe that the $\xi_{\text {opt }}$ value decreases with increasing $x$, thus leading to a decrease of residence time in the racemization reactor. On the contrary, much like $Q_{F}$, the inlet flow rate to the reactor, $Q_{r}$, increases linearly with $x$. These two opposite effects lead to a rather stable reactor volume, $V_{\mathrm{r}}$.

The conclusion of this analysis is that the IP performance does not change much with increasing $x$, while that of the SP worsens significantly. Therefore, there is no point in using values of $x$ different than zero, unless for some reasons complete separation is not attainable in the SMB unit.

Results reported in earlier literature ${ }^{39,40}$ have shown through numerical analyses that when the purity requirements are relaxed, the $\left(m_{2}, m_{3}\right)$ point moves in the corresponding plane similarly in both the linear and the nonlinear cases. On the basis of this analogy, the findings reported for the linear isotherm can be extended to nonlinear isotherm cases, for which the $m_{j}$ values need to be obtained numerically by running the full SMB model simulations. ${ }^{18}$ This was done here for a chosen isotherm, namely $\mathbf{M}_{1}$, with $k_{\mathrm{r}}^{\prime}=0.035 \mathrm{~min}^{-1}$ and $a$ $=3.3$, to verify what was expected from theoretical studies. The results reported in Figure $9 \mathrm{~b}$ agree with those of the linear isotherm, thus suggesting that the conclusions drawn above also apply to nonlinear cases.

\section{CONCLUDING REMARKS}

In the context of the production of the pure target enantiomer of a chiral compound, this work deals with the approach based on the purification of a racemic mixture obtained via symmetric synthesis. Two options have been considered, namely an integrated process, IP, combining SMB chromatography and racemization that achieves $100 \%$, and a standalone SMB process, SP, with only $50 \%$ yield.

The theoretical analysis carried out in this work is very general in scope and ambition, and is based on two important elements of novelty. On the one hand, inspired by the experimental work reported in the first part of this series, ${ }^{10}$ we 
have focused on the role of the kinetics and the conversion of the racemization reaction in determining the performance of the IP. On the other hand, by exploiting literature and experience on SMB design and optimization, we have been able to come up with an SMB optimal design, that is, both column geometry and operating conditions, that allows for a systematic comparative assessment of the two processes for broad and general system's characteristics.

In this manner we have been able to quantitatively elucidate the interplay of three elements in determining which process is better, namely the racemization kinetics, the selectivity of the chiral chromatographic method, and the burden associated with the symmetric synthesis upstream. After parametrizing these effects, we have calculated the surface separating the regions of the parameter space where one or the other process prevails in terms of productivity. Moreover, we have been able to factor in also costs considerations related to the three process elements above.

The conclusions are convincing in that they provide quantitative evidence to physical intuition. The SP is preferable, despite the $50 \%$ yield, when the racemization catalyst and the chiral stationary phase used in the SMB exhibit poor performance, and when the upstream process is relatively easy and inexpensive. When the performance of either the racemization catalyst or the chiral stationary phase improves, or better when both do, then the IP becomes more convenient; even more so as the upstream section of the process becomes more complex and more expensive. Finally, it has been shown that running the SMB under complete separation conditions appears to be always the best performing option, not only for linear and $\mathbf{M}_{\mathbf{1}}$ adsorption isotherms but also for other systems where achieving complete separation is not too difficult.

The methodology presented in this work can obviously be applied to the system studied in part $1 .{ }^{10}$ In that case the selectivity of the chiral stationary phase, $a$, is about 3 , that is, relatively high, whereas the racemization rate constant, $k_{\mathrm{r}}^{\prime}$, equals $0.035 \mathrm{~min}^{-1}$, that is, rather low. This appears to be a borderline case, in which the role of the upstream symmetric synthesis steps and of the costs of catalyst and chiral stationary phase would be decisive in determining the better process between IP and SP. Beyond the quantitative results obtained for the different cases considered in this paper, the methodology proposed and utilized here can be applied to any other chiral system of interest, thus providing a new powerful tool to the researcher and the practitioner.

\section{ASSOCIATED CONTENT}

\section{SI Supporting Information}

The Supporting Information is available free of charge at https://pubs.acs.org/doi/10.1021/acs.iecr.1c00680.

Analysis of systems with different isotherms, and different process configurations (product in the extract or raffinate), that are not included in the main manuscript for the sake of brevity; plots of the concentration as a function of selectivity for the remaining cases; physical parameter planes of the process configuration with the eutomer collected in the extract stream; cost parameter planes of the remaining isotherms of the product-in-raffinate case, as well as all the product-in-extract cases (PDF)

\section{AUTHOR INFORMATION}

\section{Corresponding Author}

Marco Mazzotti - Institute of Energy and Process Engineering, ETH Zurich, 8092 Zurich, Switzerland; (1) orcid.org/00000002-4948-6705; Phone: +41 4463224 56;

Email: marco.mazzotti@ipe.mavt.ethz.ch; Fax: +41 44632 1141

\section{Authors}

Francesca Breveglieri - Institute of Energy and Process Engineering, ETH Zurich, 8092 Zurich, Switzerland; (1) orcid.org/0000-0002-0034-7966

Tuvshinjargal Otgonbayar - Institute of Energy and Process Engineering, ETH Zurich, 8092 Zurich, Switzerland

Complete contact information is available at:

https://pubs.acs.org/10.1021/acs.iecr.1c00680

\section{Author Contributions}

${ }^{\#}$ F.B. and T.O. contributed equally to the manuscript.

\section{Notes}

The authors declare no competing financial interest.

\section{ACKNOWLEDGMENTS}

This research received funding as part of the CORE Project (October 2016-December 2020) from the European Union's Horizon 2020 Research and Innovation Program under Marie Sklodowska-Curie Grant Agreement 722456 CORE ITN.

\section{DEDICATION}

This work contributes to the collection of papers honoring Giuseppe Storti, former Professor at ETH Zurich and currently Professor at the Politecnico di Milano. With this, and with the companion part 1 of this series, the authors aim at recognizing Professor Storti's contributions to chemical engineering, particularly to adsorption and adsorption separation processes, especially to the area of simulated moving bed chromatography. Giuseppe Storti has left and is still leaving an indelible mark on all the researchers and students who have been fortunate enough to have him as collaborator, supervisor, mentor, lecturer, teacher, or even, as it is the case of the senior author of this paper, as precious friend for the last 30 years.

\section{REFERENCES}

(1) Lorenz, H.; Seidel-Morgenstern, A. Processes To Separate Enantiomers. Angew. Chem., Int. Ed. 2014, 53, 1218-1250.

(2) Oketani, R.; Hoquante, M.; Brandel, C.; Cardinael, P.; Coquerel, G. Resolution of an Atropisomeric Naphthamide by Second-Order Asymmetric Transformation: A Highly Productive Technique. Org. Process Res. Dev. 2019, 23, 1197-1203.

(3) Synoradzki, L.; Hajmowicz, H.; Wisialski, J.; Mizerski, A.; Rowicki, T. Calcium pantothenate. Part 3.1 Process for the biologically active enantiomer of the same via selective crystallization and racemization. Org. Process Res. Dev. 2008, 12, 1238-1244.

(4) Würges, K.; Petruševska-Seebach, K.; Elsner, M. P.; Lütz, S. Enzyme-assisted physicochemical enantioseparation processes - Part III: Overcoming yield limitations by dynamic kinetic resolution of asparagine via preferential crystallization and enzymatic racemization. Biotechnol. Bioeng. 2009, 104, 1235-1239.

(5) Viedma, C. Chiral Symmetry Breaking During Crystallization: Complete Chiral Purity Induced by Nonlinear Autocatalysis and Recycling. Phys. Rev. Lett. 2005, 94, 065504.

(6) Noorduin, W. L.; Vlieg, E.; Kellogg, R. M.; Kaptein, B. From Ostwald Ripening to Single Chirality. Angew. Chem., Int. Ed. 2009, 48, 9600-9606. 
(7) Suwannasang, K.; Flood, A. E.; Rougeot, C.; Coquerel, G. Using Programmed Heating-Cooling Cycles with Racemization in Solution for Complete Symmetry Breaking of a Conglomerate Forming System. Cryst. Growth Des. 2013, 13, 3498-3504.

(8) Li, W. W.; Spix, L.; de Reus, S. C. A.; Meekes, H.; Kramer, H. J. M.; Vlieg, E.; ter Horst, J. H. Deracemization of a Racemic Compound via Its Conglomerate-Forming Salt Using Temperature Cycling. Cryst. Growth Des. 2016, 16, 5563-5570.

(9) Breveglieri, F.; Maggioni, G. M.; Mazzotti, M. Deracemization of NMPA via Temperature Cycles. Cryst. Growth Des. 2018, 18, 18731881

(10) Breveglieri, F.; Otgonbayar, T.; Mazzotti, M. Optimizing the yield of a pure enantiomer by integrating chiral SMB chromatography and racemization - part 1: experiments. Ind. Eng. Chem. Res. 2021 DOI: 10.1021 /acs.iecr.1c00679

(11) Kaspereit, M.; Swernath, S.; Kienle, A. Evaluation of Competing Process Concepts for the Production of Pure Enantiomers. Org. Process Res. Dev. 2012, 16, 353-363.

(12) Fuereder, M.; Femmer, C.; Storti, G.; Panke, S.; Bechtold, M. Integration of simulated moving bed chromatography and enzymatic racemization for the production of single enantiomers. Chem. Eng. Sci. 2016, 152, 649-662.

(13) Wagner, N.; Fuereder, M.; Bosshart, A.; Panke, S.; Bechtold, M. Practical Aspects of Integrated Operation of Biotransformation and SMB Separation for Fine Chemical Synthesis. Org. Process Res. Dev. 2012, 16, 323-330.

(14) von Langermann, J.; Kaspereit, M.; Shakeri, M.; Lorenz, H.; Hedberg, M.; Jones, M. J.; Larson, K.; Herschend, B.; Arnell, R.; Temmel, E.; Bäckvall, J.-E.; Kienle, A.; Seidel-Morgenstern, A. Design of an Integrated Process of Chromatography, Crystallization and Racemization for the Resolution of 2,6-Pipecoloxylidide (PPX). Org. Process Res. Dev. 2012, 16, 343-352.

(15) Amanullah, M.; Abel, S.; Mazzotti, M. Separation of Troger's base enantiomers through a combination of simulated moving bed chromatography and crystallization. Adsorption 2005, 11, 893-897.

(16) Harriehausen, I.; Wrzosek, K.; Lorenz, H.; Seidel-Morgenstern, A. Assessment of process configurations to combine enantioselective chromatography with enzymatic racemization. Adsorption 2020, 26, 1199-1213.

(17) Kaspereit, M.; Gedicke, K.; Zahn, V.; Mahoney, A. W.; SeidelMorgenstern, A. Shortcut method for evaluation and design of a hybrid process for enantioseparations. J. Chromatogr. A 2005, 1092, 43-54.

(18) Rajendran, A.; Paredes, G.; Mazzotti, M. Simulated moving bed chromatography for the separation of enantiomers. J. Chromatogr. A 2009, 1216, 709-738.

(19) Mazzotti, M. Design of simulated moving bed separations; generalized langmuir isotherm. Ind. Eng. Chem. Res. 2006, 45, 63116324.

(20) Mazzotti, M. Equilibrium theory based design of simulated moving bed processes for a generalized Langmuir isotherm. J. Chromatogr. A 2006, 1126, 311-322.

(21) Storti, G.; Mazzotti, M.; Morbidelli, M.; Carrà, S. Robust design of binary countercurrent adsorption separation processes. AIChE J. 1993, 39, 471-492.

(22) Mazzotti, M.; Storti, G.; Morbidelli, M. Optimal operation of simulated moving bed units for nonlinear chromatographic separations. J. Chromatogr. A 1997, 769, 3-24.

(23) Juza, M.; Di Giovanni, O.; Biressi, G.; Schurig, V.; Mazzotti, M.; Morbidelli, M. Continuous enantiomer separation of the volatile inhalation anesthetic enflurane with a gas chromatographic simulated moving bed unit. J. Chromatogr. A 1998, 813, 333-347.

(24) Biressi, G.; Quattrini, F.; Juza, M.; Mazzotti, M.; Schurig, V.; Morbidelli, M. Gas chromatographic simulated moving bed separation of the enantiomers of the inhalation anesthetic enflurane. Chem. Eng. Sci. 2000, 55, 4537-4547.

(25) Juza, M.; Mazzotti, M.; Morbidelli, M. Simulated moving-bed chromatography and its application to chirotechnology. Trends Biotechnol. 2000, 18, 108-118.
(26) Jacques, J.; Collet, A.; Wilen, S. H. Enantiomers, racemates, and resolutions; J. Wiley and Sons, Inc.: Hoboken, NJ, USA, 1981.

(27) Coquerel, G. Solubility of chiral species as function of the enantiomeric excess. J. Pharm. Pharmacol. 2015, 67, 869-878.

(28) Ebbers, E. J.; Ariaans, G. J.; Houbiers, J. P.; Bruggink, A.; Zwanenburg, B. Controlled racemization of optically active organic compounds: Prospects for asymmetric transformation. Tetrahedron 1997, 53, 9417-9476.

(29) Breveglieri, F.; Mazzotti, M. Role of Racemization Kinetics in the Deracemization Process via Temperature Cycles. Cryst. Growth Des. 2019, 19, 3551-3558.

(30) Katsuo, S.; Langel, C.; Schanen, P.; Mazzotti, M. Extra-column dead volume in simulated moving bed separations: Theory and experiments. J. Chromatogr. A 2009, 1216, 1084-1093.

(31) Siitonen, J.; Mänttäri, M.; Seidel-Morgenstern, A.; Sainio, T. Robustness of steady state recycling chromatography with an integrated solvent removal unit. J. Chromatogr. A 2015, 1391, 31-39.

(32) Kaspereit, M.; Jandera, P.; Skavrada, M.; Seidel-Morgenstern, A. Impact of adsorption isotherm parameters on the performance of enantioseparation using simulated moving bed chromatography. J. Chromatogr. A 2002, 944, 249-262.

(33) Palacios, J. G.; Kaspereit, M.; Kienle, A. Integrated Simulated Moving Bed Processes for Production of Single Enantiomers. Chem. Eng. Technol. 2011, 34, 688-698.

(34) Gedicke, K.; Kaspereit, M.; Beckmann, W.; Budde, U.; Lorenz, H.; Seidel-Morgenstern, A. Conceptual Design and Feasibility Study of Combining Continuous Chromatography and Crystallization for Stereoisomer Separations. Chem. Eng. Res. Des. 2007, 85, 928-936.

(35) Storti, G.; Mazzotti, M.; Furlan, L. T.; Morbidelli, M.; Carrà, S. Performance of a Six-Port Simulated Moving-Bed Pilot Plant for Vapor-Phase Adsorption Separations. Sep. Sci. Technol. 1992, 27, $1889-1916$.

(36) Kaspereit, M.; Seidel-Morgenstern, A.; Kienle, A. Design of simulated moving bed processes under reduced purity requirements. J. Chromatogr. A 2007, 1162, 2-13.

(37) Swernath, S.; Kaspereit, M.; Kienle, A. Coupled Continuous Chromatography and Racemization Processes for the Production of Pure Enantiomers. Chem. Eng. Technol. 2014, 37, 643-651.

(38) Rajendran, A. Equilibrium theory-based design of simulated moving bed processes under reduced purity requirements. Linear isotherms. J. Chromatogr. A 2008, 1185, 216-222.

(39) Storti, G.; Baciocchi, R.; Mazzotti, M.; Morbidelli, M. Design of Optimal Operating Conditions of Simulated Moving Bed Adsorptive Separation Units. Ind. Eng. Chem. Res. 1995, 34, 288-301.

(40) Maruyama, R. T.; Karnal, P.; Sainio, T.; Rajendran, A. Design of bypass-simulated moving bed chromatography for reduced purity requirements. Chem. Eng. Sci. 2019, 205, 401-413. 